\title{
The Blueshift of the C IV Broad Emission Line in QSOs
}

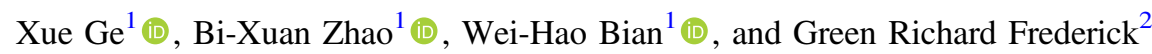 \\ ${ }^{1}$ School of Physics and Technology, Nanjing Normal University, Nanjing 210023, People’s Republic of China; whbian@njnu.edu.cn \\ ${ }^{2}$ Steward Observatory, University of Arizona, Tucson, AZ 85721, USA \\ Received 2018 November 28; revised 2019 February 2; accepted 2019 February 19; published 2019 March 20
}

\begin{abstract}
For the sample from Ge et al. of 87 low- $z$ Palomar-Green quasi-stellar objects (QSOs) and 130 high- $z$ QSOs $(0<z<5)$ with $\mathrm{H} \beta$-based single-epoch supermassive black hole masses, we perform a uniform decomposition of the C IV $\lambda 1549$ broad-line profile. Based on the rest frame defined by the [O III] $\lambda 5007$ narrow emission line, a medium-strong positive correlation is found between the C IV blueshift and the luminosity at $5100 \AA$ or the Eddington ratio $L_{\mathrm{Bol}} / L_{\mathrm{Edd}}$. A medium-strong negative relationship is found between the C IV blueshift and C IV equivalent width. These results support the postulation where the radiation pressure may be the driver of $\mathrm{C}$ IV blueshift. There is a medium-strong correlation between the mass ratio of $\mathrm{C}$ IV-based to $\mathrm{H} \beta$-based $M_{\mathrm{BH}}$ and the $\mathrm{C}$ IV blueshift, which indicates that the bias for C IV-based $M_{\mathrm{BH}}$ is affected by the C IV profile.
\end{abstract}

Key words: black hole physics - galaxies: active - quasars: emission lines

\section{Introduction}

Broad emission lines are the most prominent spectral features of Type I active galactic nuclei (AGNs) and quasi-stellar objects (QSOs). It is believed that broad emission lines are produced by photoionization. The ionizing photons are from the accretion disk surrounding the central supermassive black hole (SMBH) in AGNs/QSOs. These photons irradiate the high-velocity clouds in broad-line regions (BLRs) and the subsequent recombination produces the broad emission lines. The broad emission lines have been studied for many years with regard to their geometry and kinematics. The observed properties of broad emission lines including the width, strength, and profile can give us insight into the physical processes in the central regions of AGNs/QSOs (e.g., Wang et al. 2017).

The blueshift of the C IV emission line relative to lowionization lines is unambiguously detected in many samples (e.g., Gaskell 1982; Vanden Berk et al. 2001; Richards et al. 2002; Wang et al. 2011). It indicates that an outflowing wind may be a common configuration for the BLR clouds (Gaskell 1982; Marziani et al. 1996; Leighly 2004). Richards et al. (2011) proposed a two-component model for BLRs, namely a wind and a disk component. In this model, a stronger extreme-ultraviolet (EUV) ionizing continuum will reduce the wind component relative to the disk lines by ionizing the atoms that would otherwise produce the line-driven wind; for weaker EUV/soft X-ray, the wind component can develop, and the disk component is further reduced because the wind absorbs some of the ionizing continuum. The C IV blueshift quantifies the relative strength of these two components. The CIV equivalent width (EW) quantifies the total BLR gas and ionizing UV photons reaching the disk.

Actually, the accuracy of the C IV blueshift determination depends on the measurement of systemic redshift. There are several ways used to obtain AGNs/QSOs redshifts: (1) host galaxy absorption lines. However, these host absorption lines are usually contaminated by the light from the AGN/QSOs in spatially unresolved spectra. It makes the absorption lines too weak to be recognized. (2) The narrow [O III] or Balmer lines: these lines have been measured to have very low blueshifts or redshifts (e.g., Marziani et al. 1996; Sulentic et al. 2000; Richards et al. 2002; Hewett \& Wild 2010). In more distant
QSOs $(z>0.8)$, [O III] and $\mathrm{H} \beta$ have shifted into the nearinfrared (NIR), for which infrared spectrographs need be used to measure the common rest-frame optical lines of these QSOs. (3) UV emission lines: Mg II $\lambda 2798$ is accepted to be the most dependable UV emission line for the measurement of AGNs/ QSOs redshifts (e.g., Tytler \& Fan 1992). However, when $z>2.2$, the wavelength of $\mathrm{Mg}$ II moves beyond the optical window. CIV $\lambda 1549$ will be a reliable alternative for the measurement of redshift when $\mathrm{Mg}$ II is not available. However, the C IV outflow makes measurements of the redshifts difficult.

The $\mathrm{H} \beta$ line is widely adopted to estimate the $M_{\mathrm{BH}}$ of AGNs/ QSOs (e.g., Kaspi et al. 2000; McLure \& Jarvis 2002; Collin et al. 2006; Vestergaard \& Peterson 2006; Onken \& Kollmeier 2008; Ge et al. 2016) because of its calibration through reverberation mapping at low redshift. For the high-ionization lines, such as C IV, Baskin \& Laor (2004b) found that for QSOs with $\mathrm{H} \beta$ FWHM $<4000 \mathrm{~km} \mathrm{~s}^{-1}$, the C IV-based $M_{\mathrm{BH}}$ is higher by a factor of 3-4 than the $\mathrm{H} \beta$-based $M_{\mathrm{BH}}$. In contrast, for QSOs with $\mathrm{H} \beta \mathrm{FWHM}>4000 \mathrm{~km} \mathrm{~s}^{-1}$, the C IV-based $M_{\mathrm{BH}}$ is lower by the same factor. The C IV-based $M_{\mathrm{BH}}$ is biased with respect to the $\mathrm{H} \beta$-based $M_{\mathrm{BH}}$ (e.g., Bian et al. 2012; Shen \& Liu 2012). The uncertainties in the determination of $M_{\mathrm{BH}}$ may arise from the nonvirial component shown in the line profile, such as from a radiation-driven disk wind (e.g., Murray et al. 1995; Proga et al. 2000; Richards et al. 2011; Coatman et al. 2017).

In this paper, for a compiled sample of low- $z$ Palomar-Green (PG) QSOs and high-z QSOs, spectral decomposition is used to investigate the blueshift of the $\mathrm{C}$ IV broad emission line relative to [O III] $\lambda 5007$. The sample and analysis are described in Section 2. Our result and a discussion are given in Section 3. Finally, our conclusions are presented in Section 4. All of the cosmological calculations in this paper assume $H_{0}=$ $70 \mathrm{~km} \mathrm{~s}^{-1} \mathrm{Mpc}^{-1}, \Omega_{M}=0.3$, and $\Omega_{\Lambda}=0.7$.

\section{Sample and Analysis}

\subsection{Sample Selection}

A low- $z$ sample is adopted from the optically selected sample of PG QSOs (Boroson \& Green 1992). It contains 87 PG QSOs with $0<z<0.5$ from the Bright Quasar Survey (Schmidt \& Green 1983). It is the most thoroughly explored sample of 
Table 1

A Summary of PG QSOs

\begin{tabular}{|c|c|c|c|c|c|c|c|}
\hline (1) & $\begin{array}{l}\text { Name } \\
\text { (2) }\end{array}$ & $\begin{array}{l}z \\
(3)\end{array}$ & $\begin{array}{l}\text { Velocity Shift } \\
\left.\qquad \mathrm{km} \mathrm{s}^{-1}\right) \\
(4)\end{array}$ & $\begin{array}{l}\text { Error of Velocity Shift } \\
\qquad \begin{array}{c}\left(\mathrm{km} \mathrm{s}^{-1}\right) \\
(5)\end{array}\end{array}$ & $\begin{array}{c}L_{5100}^{\dagger} \\
\left(\operatorname{erg~s}^{-1}\right) \\
(6)\end{array}$ & $\begin{array}{c}M_{\mathrm{BH}}^{\dagger} \\
M_{\odot} \\
(7)\end{array}$ & $\begin{array}{c}L_{\mathrm{bol}} / L_{\mathrm{Edd}}^{\dagger} \\
(8)\end{array}$ \\
\hline 1 & $0003+158^{*}$ & 0.450 & 0 & 19 & 46.02 & 9.273 & -0.388 \\
\hline 2 & $0003+199^{*}$ & 0.025 & -194 & 19 & 43.72 & 7.152 & -0.566 \\
\hline 3 & $0007+106$ & 0.089 & -194 & 39 & 44.73 & 8.689 & -1.089 \\
\hline 4 & $0026+129^{*}$ & 0.142 & -968 & 19 & 44.91 & 8.594 & -0.818 \\
\hline 5 & $0049+171$ & 0.064 & -387 & 232 & 43.81 & 8.254 & -1.575 \\
\hline 6 & $0050+124^{*}$ & 0.061 & 1356 & 39 & 44.71 & 7.402 & 0.173 \\
\hline 7 & $0052+251^{*}$ & 0.155 & 775 & 39 & 44.75 & 8.567 & -0.951 \\
\hline 8 & $0157+001$ & 0.164 & 1937 & 39 & 44.91 & 8.137 & -0.360 \\
\hline 9 & $0804+761$ & 0.100 & 0 & 58 & 44.85 & 8.841 & -1.125 \\
\hline 10 & $0838+770$ & 0.131 & -387 & 174 & 44.63 & 8.110 & -0.610 \\
\hline 11 & $0844+349$ & 0.064 & -194 & 97 & 44.37 & 7.966 & -0.733 \\
\hline 12 & $0921+525$ & 0.035 & -581 & 39 & 43.59 & 7.400 & -0.944 \\
\hline 13 & $0923+129$ & 0.190 & -387 & 58 & 43.65 & 8.495 & -1.982 \\
\hline 14 & $0923+201$ & 0.029 & -968 & 136 & 44.98 & 7.984 & -0.136 \\
\hline 15 & $0947+396^{*}$ & 0.206 & 194 & 39 & 44.73 & 8.638 & -1.047 \\
\hline 16 & $0953+414^{*}$ & 0.239 & 1356 & 39 & 45.13 & 8.441 & -0.445 \\
\hline 17 & $1011-040$ & 0.058 & 0 & 77 & 44.10 & 7.243 & -0.272 \\
\hline 18 & $1012+008$ & 0.185 & 968 & 116 & 44.95 & 8.220 & -0.403 \\
\hline 19 & $1022+519$ & 0.045 & 0 & 116 & 43.46 & 7.028 & -0.706 \\
\hline 20 & $1048-090$ & 0.344 & -968 & 426 & 45.60 & 9.206 & -0.744 \\
\hline 21 & $1048+342$ & 0.167 & 581 & 329 & 44.61 & 8.324 & -0.845 \\
\hline 22 & $1049-005^{*}$ & 0.357 & 0 & 19 & 45.63 & 9.183 & -0.684 \\
\hline 23 & $1100+772^{*}$ & 0.313 & 387 & 39 & 45.58 & 9.275 & -0.834 \\
\hline 24 & $1103-006$ & 0.425 & 0 & 97 & 45.67 & 9.326 & -0.793 \\
\hline 25 & $1115+407^{*}$ & 0.154 & 581 & 77 & 44.51 & 7.616 & -0.237 \\
\hline 26 & $1116+215^{*}$ & 0.177 & 968 & 39 & 45.38 & 8.523 & -0.279 \\
\hline 27 & $1119+120$ & 0.049 & 0 & 97 & 43.96 & 7.387 & -0.561 \\
\hline 28 & $1121+422^{*}$ & 0.234 & 2518 & 19 & 44.81 & 7.996 & -0.322 \\
\hline 29 & $1149-110$ & 0.049 & 581 & 77 & 43.93 & 7.839 & -1.042 \\
\hline 30 & $1151+117$ & 0.176 & 0 & 58 & 44.67 & 8.507 & -0.975 \\
\hline 31 & $1202+281^{*}$ & 0.165 & 775 & 19 & 44.49 & 8.560 & -1.202 \\
\hline 32 & $1211+143$ & 0.085 & -194 & 39 & 45.02 & 7.938 & -0.053 \\
\hline 33 & $1216+069^{*}$ & 0.334 & 387 & 19 & 45.72 & 9.199 & -0.612 \\
\hline 34 & $1226+023^{*}$ & 0.158 & 581 & 39 & 45.90 & 8.947 & -0.181 \\
\hline 35 & $1229+204$ & 0.064 & 387 & 39 & 43.64 & 7.865 & -1.359 \\
\hline 36 & $1259+593^{*}$ & 0.472 & 1937 & 252 & 45.91 & 8.920 & -0.148 \\
\hline 37 & $1302-102^{*}$ & 0.286 & 1937 & 39 & 45.83 & 8.882 & -0.189 \\
\hline 38 & $1307+085^{*}$ & 0.155 & -194 & 39 & 44.79 & 8.643 & -0.987 \\
\hline 39 & $1310-108$ & 0.035 & -194 & 58 & 43.49 & 7.769 & -1.413 \\
\hline 40 & $1322+659^{*}$ & 0.168 & 581 & 19 & 44.92 & 8.252 & -0.469 \\
\hline 41 & $1341+258$ & 0.087 & -387 & 194 & 44.20 & 7.969 & -0.901 \\
\hline 42 & $1352+183^{*}$ & 0.158 & 2324 & 58 & 44.73 & 8.385 & -0.785 \\
\hline 43 & $1402+261^{*}$ & 0.164 & 581 & 77 & 44.92 & 7.915 & -0.129 \\
\hline 44 & $1415+451^{*}$ & 0.114 & 581 & 39 & 44.45 & 7.961 & -0.647 \\
\hline 45 & $1416-129$ & 0.129 & 0 & 58 & 45.09 & 9.025 & -1.070 \\
\hline 46 & $1425+267^{*}$ & 0.366 & 1549 & 174 & 45.76 & 9.737 & -1.110 \\
\hline 47 & $1426+015$ & 0.086 & 0 & 97 & 44.57 & 9.113 & -1.677 \\
\hline 48 & $1427+480^{*}$ & 0.221 & 0 & 19 & 44.67 & 8.046 & -0.510 \\
\hline 49 & $1435-067$ & 0.129 & 194 & 58 & 44.85 & 8.332 & -0.619 \\
\hline 50 & $1440+356^{*}$ & 0.077 & 581 & 39 & 44.43 & 7.413 & -0.117 \\
\hline 51 & $1444+407^{*}$ & 0.267 & 775 & 155 & 45.16 & 8.273 & -0.242 \\
\hline 52 & $1501+106$ & 0.036 & 194 & 58 & 44.13 & 8.451 & -1.450 \\
\hline 53 & $1512+370$ & 0.371 & 194 & 39 & 45.60 & 9.376 & -0.908 \\
\hline 54 & $1519+226$ & 0.137 & 775 & 77 & 44.62 & 7.897 & -0.416 \\
\hline 55 & $1534+580$ & 0.030 & -194 & 39 & 43.45 & 8.085 & -1.774 \\
\hline 56 & $1543+489^{*}$ & 0.400 & 2130 & 58 & 45.43 & 7.995 & 0.303 \\
\hline 57 & $1545+210^{*}$ & 0.266 & 194 & 58 & 45.41 & 9.309 & -1.030 \\
\hline 58 & $1612+261$ & 0.131 & -387 & 39 & 44.62 & 8.014 & -0.525 \\
\hline 59 & $1613+658$ & 0.129 & 581 & 77 & 44.71 & 8.446 & -0.870 \\
\hline 60 & $1617+175$ & 0.114 & 387 & 155 & 44.33 & 8.774 & -1.578 \\
\hline 61 & $1626+554$ & 0.133 & 581 & 58 & 44.47 & 8.446 & -1.111 \\
\hline 62 & $2130+099$ & 0.061 & -581 & 39 & 44.14 & 8.660 & -1.654 \\
\hline
\end{tabular}


Table 1

(Continued)

\begin{tabular}{|c|c|c|c|c|c|c|c|}
\hline (1) & $\begin{array}{l}\text { Name } \\
\text { (2) }\end{array}$ & $z$ & $\begin{array}{l}\text { Velocity Shift } \\
\left(\mathrm{km} \mathrm{s}^{-1}\right) \\
(4)\end{array}$ & $\begin{array}{l}\text { Error of Velocity Shift } \\
\qquad \begin{array}{c}\left(\mathrm{km} \mathrm{s}^{-1}\right) \\
(5)\end{array}\end{array}$ & $\begin{array}{c}L_{5100}^{\dagger} \\
\left(\mathrm{erg} \mathrm{s}^{-1}\right) \\
(6)\end{array}$ & $\begin{array}{c}M_{\mathrm{BH}}^{\dagger} \\
M_{\odot} \\
(7)\end{array}$ & $\begin{array}{c}L_{\mathrm{bol}} / L_{\text {Edd }}^{\dagger} \\
\text { (8) }\end{array}$ \\
\hline 63 & $2209+184$ & 0.070 & 775 & 174 & 44.34 & 8.706 & -1.496 \\
\hline 64 & $2214+139$ & 0.067 & 0 & 97 & 44.56 & 8.503 & -1.076 \\
\hline 65 & $2251+113^{*}$ & 0.323 & -387 & 39 & 45.69 & 8.992 & -0.433 \\
\hline 66 & $2304+042$ & 0.042 & 0 & 58 & 43.88 & 8.476 & -1.726 \\
\hline 67 & $2308+098^{*}$ & 0.432 & -194 & 39 & 45.78 & 9.595 & -0.952 \\
\hline
\end{tabular}

Note. Column (1): sequence number; column (2): name of PG QSOs, * in column (2) indicate the observations that are from HST; column (3): redshift from Schmidt \& Green (1983); columns (4) and (5): shift of C IV peak wavelength and the error, positive value means blueshift; column (6): luminosity of 5100 Aं; columns (7) and (8): the host-corrected $M_{\mathrm{BH}}$ and Eddington ratio (in logarithm scale). $\dagger$ means that the data is from Ge et al. (2016).

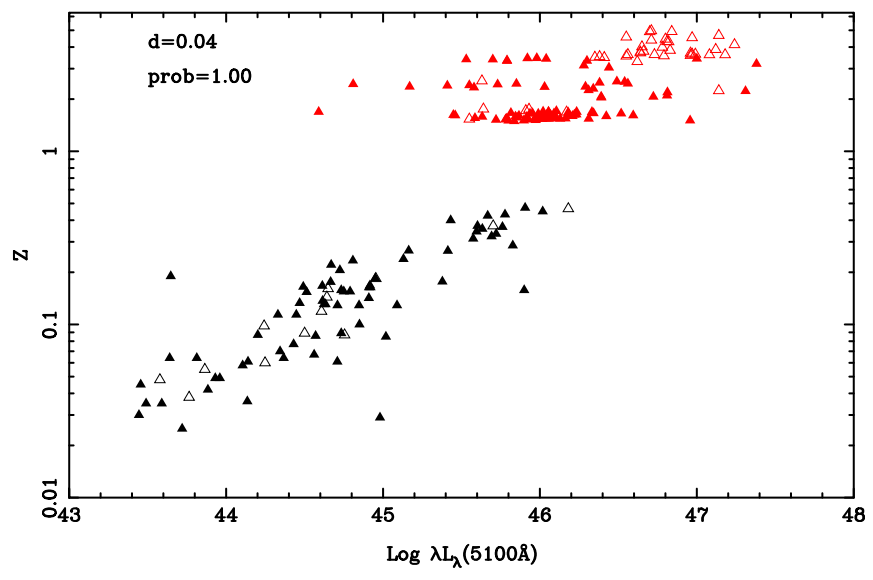

Figure 1. Distribution of low-z QSOs (black) and high- $z$ QSOs (red) in $z-\lambda L_{\lambda}(5100 \AA)$ space.The hollow triangles are excluded from our sample. The labels in the panel are the two-dimension K-S test.

AGN/QSO, with a lot of high-quality broadband data, including X-ray, optical, infrared, and radio (e.g., Boroson \& Green 1992; Brandt et al. 2000; Baskin \& Laor 2004a, 2004b; Shi et al. 2014; Bian et al. 2016). The optical spectra of the 87 objects are from the Gold Spectrograph on the Kitt Peak National Observatory (KPNO) $2.1 \mathrm{~m}$ telescope. The resolution of the spectra is $6.5 \AA$ corresponding to $\sim 400 \mathrm{~km} \mathrm{~s}^{-1}$. The redshift of each QSO was measured from the [O III] $\lambda 5007$ narrow emission line except for eight QSOs with weak or absent [O III]. Their redshifts were measured through the $\mathrm{H} \beta$ line (Boroson \& Green 1992).

The UV spectra covering C IV $1549 \AA$ of 85 PG QSOs are available in the MAST archive, 47 from the Hubble Space Telescope (HST), and 38 from the International Ultraviolet Explorer (IUE). Three sources, PG 0934+013, PG 1004+130, and PG $1448+273$, do not have enough signal-to-noise ratio $(\mathrm{S} / \mathrm{N})$ to fit their $\mathrm{C}$ IV profiles. We also exclude 15 sources that are heavily absorbed in the C IV region (Laor \& Brandt 2002; Baskin \& Laor 2004b) because our fitting method used here is not suitable for profiles with a broad absorption line (BAL). Finally, there are 67 PG QSOs which are available for spectral decomposition. The UV spectra for 35 PG QSOs are available from $H S T$, which were observed with the Faint Object Spectrograph (FOS) covering the wavelength range of $1150-8500 \AA$. The spectral resolutions $(R=\lambda / \Delta \lambda)$ for FOS observations are $R \approx 1300$ and $R \approx 250$ at high and low spectral resolution, respectively. The UV spectra for the $32 \mathrm{PG}$ QSOs are available from $I U E$ with a spectral resolution of
$R \approx 200$ (Anand et al. 2009). The wavelength range of the $I U E$ UV spectra are 1151-2000 $\AA$ and 1850-3400 $\AA$ at shortwavelength prime (SWP) and long-wavelength prime (LWP), respectively. For some PG QSOs with IUE spectra, we did not add other observations, although a small number of sources were observed again using other spectrographs. We think that the choice of spectrographs of will not impact our results. Table 1 lists the information for these 67 PG QSOs.

The Sloan Digital Sky Survey (SDSS) I/II data (data release from 1 to 7, DR1-DR7) are available from the dedicated widefield $2.5 \mathrm{~m}$ telescope (Gunn et al. 2006) at Apache Point Observatory near Sacramento Peak in southern New Mexico. The spectral resolution is up to $69 \mathrm{~km} \mathrm{~s}^{-1}$. The wavelength range is from $3800 \AA$ to $9200 \AA$. SDSS III (data release from 8 to 12, DR8-DR12) extends the range of wavelength from $3600 \AA$ to $10400 \AA$. Ge et al. (2016) collected 181 high-z QSOs with $\mathrm{H} \beta$-based $M_{\mathrm{BH}}$ from different literatures. They crossmatched the high- $z$ sources with Shen et al. (2011) and obtained only 125 sources with C IV fitting data. ${ }^{3}$ In this paper, we use 130 out of 181 sources where 125 objects are from Ge et al. (2016) and their UV spectra are obtained from SDSS DR7 (Abazajian et al. 2009; York et al. 2000). For 5 of the 130 high$z$ QSOs (i.e., TON618, UM667, SDSSJ105123+35453, SDSSJ153650+50081, and SDSSJ165354+40540), their UV spectra are from SDSS DR12 (Pâris et al. 2016) because the UV spectra with C IV are not available in SDSS DR7.

To get reliable measurements of the blueshift of the $\mathrm{C}$ IV line, we constructed a subsample from these low $z$ and high- $z$ QSOs, i.e., 67 PG QSOs and 112 high-z QSOs. For high- $z$ QSOs, we eliminated nine BAL QSOs identified by Shen et al. (2011) and visualizing, and seven weak-line sources from Shemmer \& Lieber (2015). In addition, we also excluded Q0142-100 and PG 1115+080 from Assef et al. (2011), because of the lack of a redshift estimate from [O III] or $\mathrm{H} \beta / \mathrm{H} \alpha$. The high- $z$ QSOs in the subsample comprise 112 QSOs, where 60 are from Shen \& Liu (2012), 3 are from Assef et al. (2011), 12 are from Shemmer et al. (2004), 15 are from Netzer et al. (2007), and 22 are from Jun et al. (2015). We perform the two-dimensional KolmogorovSmirnov (K-S) test on the entire sample and subsample and find no significant difference between the two samples (Figure 1). The large $\mathrm{p}$ value $(>0.001)$ shows that our selection of subsample does not significantly affect the results of this work. We call the subsample as "sample," which will be analyzed in following sections. Table 2 lists the information for these high-z QSOs.

\footnotetext{
3 SDSS081331+254503 and HS0810+2554 in their Table 2 are actually the same.
} 
Table 2

A Summary of High-z QSOs

\begin{tabular}{|c|c|c|c|c|c|c|c|c|}
\hline (1) & $\begin{array}{l}\text { Name } \\
\text { (2) }\end{array}$ & $\begin{array}{l}z_{\text {fit }} \\
\text { (3) }\end{array}$ & $\begin{array}{l}\text { Velocity Shift } \\
\left(\mathrm{km} \mathrm{s}^{-1}\right) \\
(4)\end{array}$ & $\begin{array}{l}\text { Error of Velocity Shift } \\
\qquad \begin{array}{c}\left(\mathrm{km} \mathrm{s}^{-1}\right) \\
(5)\end{array}\end{array}$ & $\begin{array}{l}L_{5100}^{\dagger} \\
\left(\mathrm{erg} \mathrm{s}^{-1}\right) \\
(6)\end{array}$ & $\begin{array}{c}M_{\mathrm{BH}}^{\dagger} \\
M_{\odot} \\
(7)\end{array}$ & $\begin{array}{c}L_{\text {bol }} / L_{\text {Edd }}^{\dagger} \\
\quad(8)\end{array}$ & $\begin{array}{c}\text { Reference } \\
\text { (9) }\end{array}$ \\
\hline 1 & SDSS225800.02-084143 & 1.496 & 793 & 80 & 45.836 & 8.835 & -0.129 & $\mathrm{a}$ \\
\hline 2 & SDSS035856.73-054023 & 1.506 & 649 & 133 & 45.800 & 9.065 & -0.395 & $\mathrm{a}$ \\
\hline 3 & SDSS133321.90+005824 & 1.511 & -152 & 30 & 45.897 & 9.391 & -0.624 & $\mathrm{a}$ \\
\hline 4 & SDSS081331.28+254503 & 1.513 & 14 & 19 & 46.957 & 9.802 & 0.025 & $\mathrm{a}$ \\
\hline 5 & SDSS152111.86+470539 & 1.517 & 71 & 25 & 45.973 & 9.312 & -0.469 & $\mathrm{a}$ \\
\hline 6 & FBQ1633+3134 & 1.522 & 519 & 185 & 45.720 & 9.096 & -0.506 & $\mathrm{~b}$ \\
\hline 7 & SDSS085543.26+002908 & 1.525 & 303 & 30 & 45.781 & 9.284 & -0.633 & $\mathrm{a}$ \\
\hline 8 & SDSS123355.21+031327 & 1.528 & 904 & 71 & 45.927 & 9.591 & -0.794 & $\mathrm{a}$ \\
\hline 9 & SDSS104910.31+143227 & 1.540 & 776 & 29 & 46.013 & 9.036 & -0.153 & $\mathrm{a}$ \\
\hline 10 & SDSS154212.90+111226 & 1.540 & 1500 & 106 & 46.055 & 9.498 & -0.573 & $\mathrm{a}$ \\
\hline 11 & SDSS143230.57+012435 & 1.542 & 1764 & 67 & 45.966 & 8.755 & 0.081 & $\mathrm{a}$ \\
\hline 12 & SDSS074029.82+281458 & 1.545 & 1263 & 36 & 46.041 & 9.514 & -0.603 & $\mathrm{a}$ \\
\hline 13 & SDSS081344.15+152221 & 1.545 & 1391 & 75 & 46.031 & 9.391 & -0.490 & $\mathrm{a}$ \\
\hline 14 & SDSS082146.22+571226 & 1.546 & 1225 & 49 & 46.311 & 9.429 & -0.248 & $\mathrm{a}$ \\
\hline 15 & SDSS171030.20+602347 & 1.549 & -443 & 54 & 46.129 & 9.655 & -0.656 & $\mathrm{a}$ \\
\hline 16 & SDSS123442.16+052126 & 1.550 & 985 & 52 & 46.163 & 9.816 & -0.783 & $\mathrm{a}$ \\
\hline 17 & SDSS015733.87-004824 & 1.551 & 497 & 162 & 45.786 & 9.352 & -0.696 & $\mathrm{a}$ \\
\hline 18 & SDSS101447.54+521320 & 1.552 & 396 & 54 & 46.016 & 9.015 & -0.129 & $\mathrm{a}$ \\
\hline 19 & SDSS135439.70+301649 & 1.553 & 600 & 45 & 46.097 & 9.465 & -0.498 & $\mathrm{a}$ \\
\hline 20 & SDSS223246.80+134702 & 1.557 & -254 & 34 & 46.060 & 9.762 & -0.832 & $\mathrm{a}$ \\
\hline 21 & SDSS100930.51+023052 & 1.557 & 42 & 52 & 45.586 & 9.051 & -0.595 & $\mathrm{a}$ \\
\hline 22 & SDSS124006.70+474003 & 1.561 & 644 & 40 & 45.978 & 8.864 & -0.016 & $\mathrm{a}$ \\
\hline 23 & SDSS093318.49+141340 & 1.561 & 246 & 53 & 46.099 & 9.649 & -0.680 & $\mathrm{a}$ \\
\hline 24 & SDSS094126.49+044328 & 1.567 & 394 & 44 & 45.854 & 9.455 & -0.731 & $\mathrm{a}$ \\
\hline 25 & SDSS113829.33+040101 & 1.567 & 1527 & 55 & 46.086 & 9.937 & -0.981 & $\mathrm{a}$ \\
\hline 26 & SDSS084451.91+282607 & 1.574 & 1513 & 29 & 45.916 & 8.698 & 0.088 & $\mathrm{a}$ \\
\hline 27 & SDSS081227.19+075732 & 1.574 & 341 & 72 & 46.002 & 9.895 & -1.023 & $\mathrm{a}$ \\
\hline 28 & SDSS091754.44+043652 & 1.587 & -214 & 31 & 45.632 & 9.664 & -1.162 & $\mathrm{a}$ \\
\hline 29 & SDSS204538.96-005115 & 1.590 & 428 & 56 & 45.844 & 9.180 & -0.466 & $\mathrm{a}$ \\
\hline 30 & SDSS014705.42+133210 & 1.595 & 1340 & 40 & 46.207 & 9.321 & -0.244 & $\mathrm{a}$ \\
\hline 31 & SDSS114023.40+301651 & 1.599 & 1462 & 43 & 46.423 & 9.421 & -0.128 & $\mathrm{a}$ \\
\hline 32 & SDSS104603.22+112828 & 1.607 & 444 & 44 & 45.866 & 9.200 & -0.464 & $\mathrm{a}$ \\
\hline 33 & SDSS125140.82+080718 & 1.607 & 1681 & 97 & 46.091 & 9.016 & -0.055 & $\mathrm{a}$ \\
\hline 34 & SDSS204009.62-065402 & 1.611 & 733 & 97 & 45.460 & 8.997 & -0.667 & $\mathrm{a}$ \\
\hline 35 & SDSS155240.40+194816 & 1.613 & 1183 & 60 & 46.044 & 9.647 & -0.733 & $\mathrm{a}$ \\
\hline 36 & SDSS002948.04-095639 & 1.618 & 156 & 24 & 46.022 & 8.923 & -0.031 & $\mathrm{a}$ \\
\hline 37 & SDSS083850.15+261105 & 1.618 & 421 & 19 & 46.595 & 9.420 & 0.045 & $\mathrm{a}$ \\
\hline 38 & SDSS205554.08+004311 & 1.624 & 827 & 78 & 45.448 & 8.957 & -0.639 & $\mathrm{a}$ \\
\hline 39 & SDSS135023.68+265243 & 1.624 & 1132 & 36 & 46.219 & 9.182 & -0.093 & $\mathrm{a}$ \\
\hline 40 & SDSS111949.30+233249 & 1.626 & 231 & 21 & 46.067 & 9.558 & -0.621 & $\mathrm{a}$ \\
\hline 41 & SDSS004149.64-094705 & 1.629 & 571 & 46 & 46.187 & 9.636 & -0.579 & $\mathrm{a}$ \\
\hline 42 & SDSS160456.14-001907 & 1.636 & 1272 & 70 & 46.232 & 9.435 & -0.333 & $\mathrm{a}$ \\
\hline 43 & SDSS141949.39+060654 & 1.649 & 2297 & 62 & 45.936 & 9.319 & -0.513 & $\mathrm{a}$ \\
\hline 44 & SDSS020044.50+122319 & 1.654 & 148 & 40 & 45.989 & 9.260 & -0.401 & $\mathrm{a}$ \\
\hline 45 & SDSS142841.97+592552 & 1.660 & 352 & 53 & 46.089 & 9.179 & -0.220 & $\mathrm{a}$ \\
\hline 46 & SDSS204536.56-010147 & 1.661 & 252 & 35 & 46.518 & 9.789 & -0.401 & $\mathrm{a}$ \\
\hline 47 & SDSS110240.16+394730 & 1.664 & 1591 & 71 & 45.953 & 9.333 & -0.510 & $\mathrm{a}$ \\
\hline 48 & SDSS100401.27+423123 & 1.666 & 1412 & 194 & 46.342 & 9.280 & -0.068 & $\mathrm{a}$ \\
\hline 49 & SDSS213748.44+001220 & 1.670 & 79 & 47 & 45.815 & 9.056 & -0.371 & $\mathrm{a}$ \\
\hline 50 & SDSS094913.05+175155 & 1.675 & 2361 & 85 & 46.180 & 9.493 & -0.443 & $\mathrm{a}$ \\
\hline 51 & SDSS153859.45+053705 & 1.684 & 975 & 34 & 46.055 & 9.004 & -0.079 & $\mathrm{a}$ \\
\hline 52 & SDSS162103.98+002905 & 1.689 & 2539 & 166 & 46.020 & 9.452 & -0.562 & $\mathrm{a}$ \\
\hline 53 & SDSS0246-0825 & 1.690 & 693 & 51 & 44.590 & 8.001 & -0.541 & $\mathrm{~b}$ \\
\hline 54 & SDSS105951.05+090905 & 1.690 & 172 & 10 & 46.331 & 9.402 & -0.201 & $\mathrm{a}$ \\
\hline 55 & SDSS041255.16-061210 & 1.691 & 1589 & 77 & 46.054 & 9.256 & -0.332 & $\mathrm{a}$ \\
\hline 56 & SDSS112542.29+000101 & 1.692 & -111 & 12 & 46.234 & 9.298 & -0.194 & $\mathrm{a}$ \\
\hline 57 & SDSS101504.75+123022 & 1.703 & 3212 & 125 & 46.105 & 9.298 & -0.323 & $\mathrm{a}$ \\
\hline 58 & SDSS122039.45+000427 & 2.048 & 870 & 29 & 46.391 & 9.230 & 0.031 & $\mathrm{a}$ \\
\hline 59 & SDSS143645.80+633637 & 2.066 & 398 & 17 & 46.723 & 9.969 & -0.376 & $\mathrm{a}$ \\
\hline 60 & SDSS014944.43+150106 & 2.073 & 994 & 22 & 46.390 & 9.657 & -0.397 & $\mathrm{a}$ \\
\hline 61 & SDSS143148.09+053558 & 2.095 & 863 & 22 & 46.810 & 10.561 & -0.881 & $\mathrm{a}$ \\
\hline 62 & SDSS142108.71+224117 & 2.188 & 976 & 17 & 46.813 & 9.868 & -0.185 & $\mathrm{a}$ \\
\hline
\end{tabular}


Table 2

(Continued)

\begin{tabular}{|c|c|c|c|c|c|c|c|c|}
\hline (1) & $\begin{array}{l}\text { Name } \\
\text { (2) }\end{array}$ & $\begin{array}{l}z_{\text {fit }} \\
\text { (3) }\end{array}$ & $\begin{array}{l}\text { Velocity Shift } \\
\qquad \begin{array}{c}\left(\mathrm{km} \mathrm{s}^{-1}\right) \\
(4)\end{array}\end{array}$ & $\begin{array}{l}\text { Error of Velocity Shift } \\
\qquad \begin{array}{c}\left(\mathrm{km} \mathrm{s}^{-1}\right) \\
(5)\end{array}\end{array}$ & $\begin{array}{c}L_{5100}^{\dagger} \\
\left(\mathrm{erg} \mathrm{s}^{-1}\right) \\
(6)\end{array}$ & $\begin{array}{c}M_{\mathrm{BH}}^{\dagger} \\
M_{\odot} \\
(7)\end{array}$ & $\begin{array}{c}L_{\text {bol }} / L_{\text {Edd }}^{\dagger} \\
\text { (8) }\end{array}$ & $\begin{array}{c}\text { Reference } \\
\text { (9) }\end{array}$ \\
\hline 63 & TON618 & 2.219 & 2761 & 423 & 47.310 & 10.610 & -0.430 & $\mathrm{c}$ \\
\hline 64 & UM645 & 2.268 & -644 & 54 & 46.310 & 9.458 & -0.278 & $\mathrm{c}$ \\
\hline 65 & SDSSJ170102.18+61230 & 2.290 & 2474 & 110 & 46.340 & 9.601 & -0.391 & $\mathrm{c}$ \\
\hline 66 & SDSSJ115111.20+03404 & 2.337 & 74 & 504 & 45.580 & 9.123 & -0.673 & $\mathrm{~d}$ \\
\hline 67 & SDSSJ144245.66-02425 & 2.355 & 1360 & 144 & 46.030 & 9.052 & -0.152 & $\mathrm{~d}$ \\
\hline 68 & SDSSJ100710.70+04211 & 2.367 & 675 & 121 & 45.170 & 8.978 & -0.938 & $\mathrm{~d}$ \\
\hline 69 & UM642 & 2.372 & 83 & 69 & 46.290 & 9.532 & -0.372 & $\mathrm{c}$ \\
\hline 70 & SDSSJ125034.41-01051 & 2.399 & 378 & 62 & 45.410 & 9.038 & -0.758 & $\mathrm{~d}$ \\
\hline 71 & SDSSJ095141.33+01325 & 2.419 & -599 & 33 & 45.550 & 8.951 & -0.531 & d \\
\hline 72 & SDSSJ101257.52+02593 & 2.441 & 795 & 103 & 45.730 & 8.955 & -0.355 & d \\
\hline 73 & SDSS1138+0314 & 2.445 & 250 & 20 & 44.810 & 8.504 & -0.824 & $\mathrm{~b}$ \\
\hline 74 & SDSSJ025438.37+00213 & 2.464 & -121 & 49 & 45.850 & 9.074 & -0.354 & d \\
\hline 75 & UM629 & 2.471 & 709 & 30 & 46.560 & 8.993 & 0.437 & $\mathrm{c}$ \\
\hline 76 & SDSSJ024933.42-08345 & 2.494 & 277 & 20 & 46.380 & 9.537 & -0.287 & $\mathrm{c}$ \\
\hline 77 & SDSSJ135445.66+00205 & 2.514 & 3126 & 179 & 46.490 & 8.994 & 0.366 & $\mathrm{c}$ \\
\hline 78 & UM632 & 2.521 & 85 & 20 & 46.540 & 9.346 & 0.064 & c \\
\hline 79 & SDSSJ100428.43+00182 & 3.054 & 399 & 25 & 46.440 & 9.204 & 0.106 & $\mathrm{c}$ \\
\hline 80 & UM667 & 3.122 & 422 & 71 & 46.280 & 9.042 & 0.108 & $\mathrm{c}$ \\
\hline 81 & SBS1425+606 & 3.197 & 2207 & 44 & 47.380 & 10.261 & -0.011 & c \\
\hline 82 & SDSSJ083700.82+35055 & 3.316 & 14 & 22 & 46.620 & 9.835 & -0.345 & $\mathrm{e}$ \\
\hline 83 & SDSSJ210258.21+00202 & 3.342 & -457 & 42 & 45.790 & 9.519 & -0.859 & d \\
\hline 84 & SDSSJ210311.69-06005 & 3.344 & 511 & 38 & 46.300 & 9.627 & -0.457 & d \\
\hline 85 & SDSSJ113838.26-02060 & 3.347 & 1406 & 187 & 45.790 & 9.123 & -0.463 & $\mathrm{~d}$ \\
\hline 86 & SDSSJ083630.55+06204 & 3.384 & 1796 & 219 & 45.530 & 8.868 & -0.468 & d \\
\hline 87 & SDSSJ105511.99+02075 & 3.404 & 562 & 67 & 45.700 & 9.229 & -0.659 & $\mathrm{~d}$ \\
\hline 88 & SDSSJ173352.22+54003 & 3.435 & 662 & 39 & 47.000 & 9.387 & 0.483 & $\mathrm{c}$ \\
\hline 89 & SDSSJ115304.62+03595 & 3.437 & 43 & 49 & 46.040 & 9.414 & -0.504 & d \\
\hline 90 & SDSSJ115935.64+04242 & 3.456 & 213 & 39 & 45.920 & 9.360 & -0.570 & $\mathrm{~d}$ \\
\hline 91 & SDSSJ153725.36-01465 & 3.467 & 626 & 101 & 45.980 & 9.026 & -0.176 & d \\
\hline 92 & SDSSJ164248.71+24030 & 3.497 & 710 & 75 & 46.410 & 8.911 & 0.369 & e \\
\hline 93 & SDSSJ150620.48+46064 & 3.503 & 1784 & 124 & 46.380 & 9.788 & -0.538 & $\mathrm{e}$ \\
\hline 94 & SDSSJ075303.33+42313 & 3.595 & -2068 & 12 & 46.790 & 9.895 & -0.235 & $\mathrm{e}$ \\
\hline 95 & SDSSJ144144.76+47200 & 3.643 & -212 & 31 & 46.560 & 9.097 & 0.333 & $\mathrm{e}$ \\
\hline 96 & SDSSJ145408.95+51144 & 3.648 & -1418 & 29 & 47.080 & 9.790 & 0.160 & $\mathrm{e}$ \\
\hline 97 & SDSSJ101336.37+56153 & 3.652 & 1357 & 353 & 46.990 & 10.051 & -0.191 & $\mathrm{e}$ \\
\hline 98 & SDSSJ014049.18-08394 & 3.726 & -467 & 38 & 46.960 & 9.797 & 0.033 & $\mathrm{e}$ \\
\hline 99 & SDSSJ113307.63+52283 & 3.744 & 1742 & 645 & 46.640 & 9.026 & 0.484 & $\mathrm{e}$ \\
\hline 100 & SDSSJ162520.31+22583 & 3.774 & 1433 & 47 & 46.660 & 9.700 & -0.170 & $\mathrm{e}$ \\
\hline 101 & SDSSJ012403.77+00443 & 3.827 & 1309 & 104 & 46.830 & 9.475 & 0.225 & $\mathrm{e}$ \\
\hline 102 & SDSSJ144542.75+49024 & 3.881 & -221 & 17 & 47.120 & 10.105 & -0.115 & $\mathrm{e}$ \\
\hline 103 & SDSSJ132420.83+42255 & 4.042 & -630 & 100 & 46.650 & 9.280 & 0.240 & $\mathrm{e}$ \\
\hline 104 & SDSSJ105756.28+45555 & 4.130 & 324 & 79 & 47.240 & 9.781 & 0.329 & $\mathrm{e}$ \\
\hline 105 & SDSSJ095511.32+59403 & 4.364 & -2408 & 52 & 46.810 & 9.454 & 0.226 & $\mathrm{e}$ \\
\hline 106 & SDSSJ083946.22+51120 & 4.408 & -456 & 214 & 46.710 & 9.853 & -0.273 & $\mathrm{e}$ \\
\hline 107 & SDSSJ010619.24+00482 & 4.450 & 380 & 37 & 46.800 & 10.089 & -0.419 & $\mathrm{e}$ \\
\hline 108 & SDSSJ134743.29+49562 & 4.536 & 881 & 282 & 46.970 & 10.057 & -0.217 & $\mathrm{e}$ \\
\hline 109 & SDSSJ163636.92+31571 & 4.570 & 1314 & 339 & 46.550 & 9.832 & -0.412 & $\mathrm{e}$ \\
\hline 110 & SDSSJ143835.95+43145 & 4.669 & 645 & 168 & 47.140 & 9.864 & 0.146 & $\mathrm{e}$ \\
\hline 111 & SDSSJ105123.03+35453 & 4.924 & -111 & 36 & 46.700 & 9.634 & -0.064 & $\mathrm{e}$ \\
\hline 112 & SDSSJ153650.25+50081 & 4.941 & 258 & 15 & 46.840 & 9.592 & 0.118 & $\mathrm{e}$ \\
\hline
\end{tabular}

Note. Column (1): sequence number; column (2): name of high-z QSOs; column (3): redshift used to fit spectrum; columns (4) and (5): shift of the C IV peak wavelength and the error, where the positive value means blueshift; column (6): the luminosity of $5100 \AA$; columns (7) and (8): $M_{\mathrm{BH}}$ and Eddington ratio (in logarithm scale); column (9): references. (a) Shen \& Liu (2012), (b) Assef et al. (2011), (c) Shemmer et al. (2004), (d) Netzer et al. (2007), and (e) Jun et al. (2015). $\dagger$ means that the data is from Ge et al. (2016) and the fitting redshifts of UM667 and TON618 are from the NASA/IPAC Extragalactic Database.

The high- $z$ sources were chosen with high $\mathrm{S} / \mathrm{N}$ in SDSS spectra, have $\mathrm{H} \beta /[\mathrm{O}$ III] , and fall in a good place in the NIR atmospheric window. The high $\mathrm{S} / \mathrm{N}$, however, may lead the QSOs with very broad lines and low EW in CIV to be underrepresented in this work. One property of our sample is that PG and high- $z$ QSOs are bright in the UV band. This property reduces the objects that are reddened by circumnuclear dust. The dust might differentially suppress some of the C IV emitting material and the dust-obscured objects may lead a different correlation, but would not be as valid for learning 

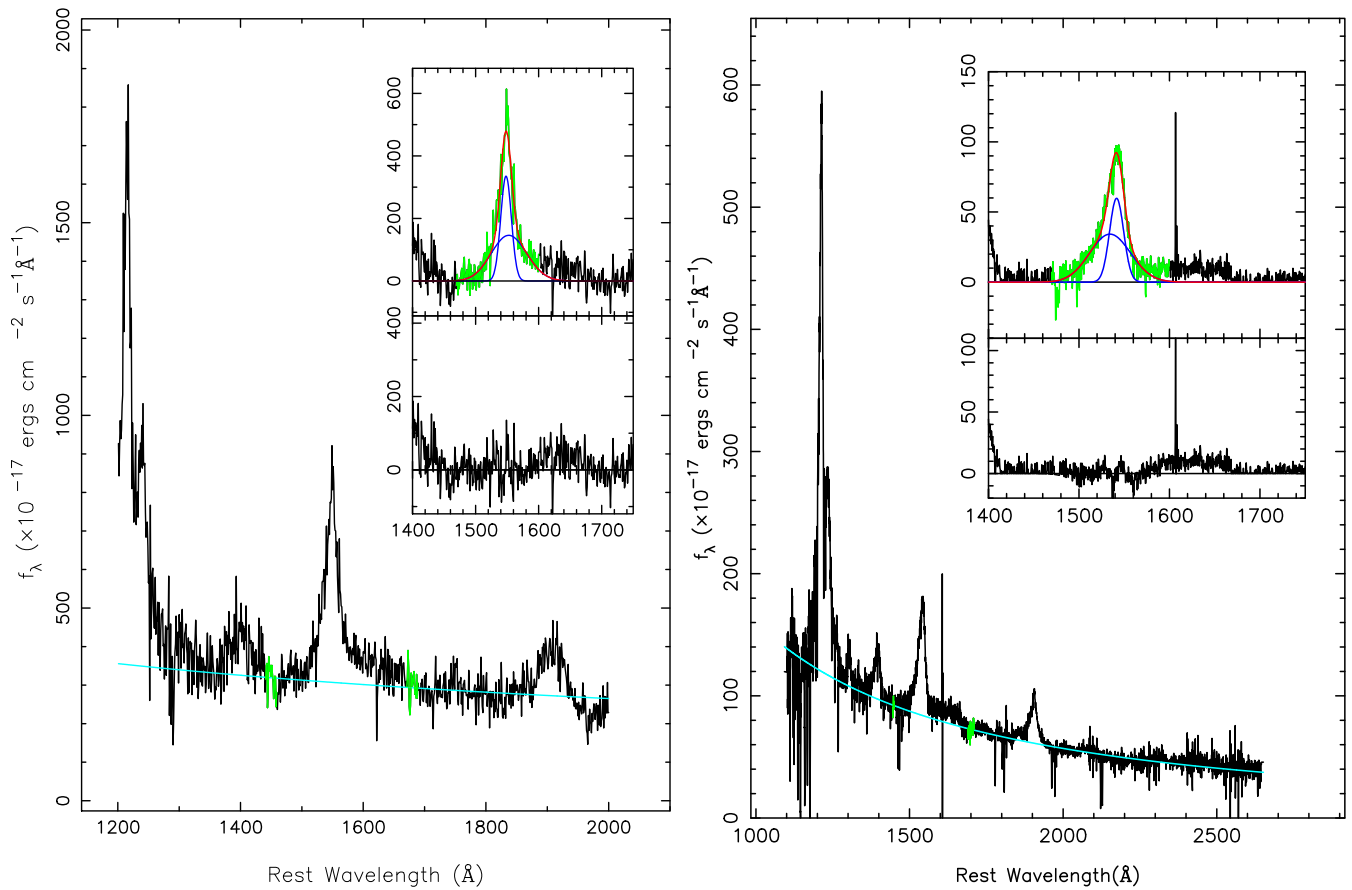

Figure 2. Examples of the UV continuum and emission line fittings for a low- $z$ (left) and a high- $z$ (right) QSO. The original spectra and the fitting windows for the continuum are marked in black and green. The cyan curve is the power law that represents the continuum. The inset shows the fitting of the C IV emission line. The fitting window is also shown in green. The narrow and broad components are marked in blue, and their sum is marked in red. The residual spectrum is shown at the bottom.

about the physical origin of the total C IV profile. It is worth emphasizing that the $M_{\mathrm{BH}}$ of the sample is $\mathrm{H} \beta$-based thanks to the infrared observation. This unity enables us to explore the relationship between the CIV blueshift and $L_{\mathrm{Bol}} / L_{\mathrm{Edd}}$ from low- $z$ to high- $z$ QSOs. In addition, There are 14 radio-loud QSOs in PG QSOs and 11 high-z QSOs, respectively. We do not exclude these objects and will discuss their C IV blueshift in Section 3.

\subsection{UV Spectral Decomposition}

We need to point out that an average spectrum, weighted by the $\mathrm{S} / \mathrm{N}$, is adopted when more than one spectrum is available. We performed least $-\chi^{2}$ fits to the UV emission line spectra. The $\chi^{2}$ is determined by the error in the flux. The mean reduced $\chi^{2}$ are 1.43 and 1.25 for high $z$ and low- $z$ QSOs, respectively. We list the steps of spectral decomposition as follows. First, the spectra are corrected for Galactic extinction using their $A_{V}$ values from the NASA/IPAC Extragalactic Database assuming an extinction curve with $R_{V}=3.1$ (Cardelli et al. 1989). The extinction-corrected observed spectra are then transformed to the rest frame; no extinction correction is applied for the host galaxy. For PG QSOs, we use the redshifts from Boroson \& Green (1992). For high- $z$ QSOs, we adopt the redshifts from Hewett \& Wild (2010). For UM667 and TON618, their redshifts are adopted from the NASA/IPAC Extragalactic Database because their redshifts are not available in Hewett \& Wild (2010). Next, we fit the continuum using a power-law function in two fitting windows near $\sim 1450 \AA$ and $\sim 1710 \AA$. Finally, the C IV emission line in the continuum-subtracted spectrum is fitted with two Gaussians, one with intermediate velocity width and another with broader width. The FWHM of the two components from BLRs is restricted to be more than 1000 and less than $20,000 \mathrm{~km} \mathrm{~s}^{-1}$. Simultaneously, we constrain the centers of their profiles to have a shift range of $\sim 15 \AA$. The fitting window for the $\mathrm{C}$ IV line covers the range of 1470-1600 $\AA$, considering the contamination of He II $\lambda 1640$. It is found that a two-Gaussian fit is adequate for the C IV profile in our low- $z$ and high $z$ QSOs. Figure 2 shows the examples of the fitting of the continuum and the $\mathrm{C}$ IV line. We list the profile parameters of low- and high- $z$ QSOs in Tables 3 and 4.

Wills et al. (1993) and Brotherton et al. (1994) presented a statistical investigation of broad emission-line profiles (such as C IV, Mg II) in QSOs. They proposed a two-component model with a narrow core component (FWHM $\sim 2000 \mathrm{~km} \mathrm{~s}^{-1}$ ) and a broad base component (FWHM $\sim 7000 \mathrm{~km} \mathrm{~s}^{-1}$ ) with a blueshift of $\sim 1000 \mathrm{~km} \mathrm{~s}^{-1}$ relative to the core component to interpret their results. They suggested that the core-to-base ratio determines the profile of the emission line.

\subsection{Blueshift, $M_{B H}$, and $L_{B o l} / L_{E d d}$}

For the two-Gaussian fitting of the C IV line, the EW(C IV) of the entire profile is calculated by integrating both components and the results are consistent with $\mathrm{Wu}$ et al. (2009). The error for EW(C IV) is derived from the errors of the integrated C IV flux and the continuum flux in our fits.

From the reconstructed C IV profile of our two-Gaussian fitting, the C IV peak wavelength is calculated as well as its error. With respect to the rest frame defined by the [O III] emission line or the Balmer emission line (i.e., $z_{\mathrm{sys}}$ ), the C IV blueshift and the error can be calculated following Equations (1) and (2), where $c$ is the speed of light. In Equation (1), $\lambda$ is the C IV peak wavelength in the rest frame defined by $z_{\text {fit }}$ used in our fitting, and $z_{\text {sys }}$ is the systemic redshift that is defined by the [O III] or Balmer emission line. The error of the C IV blueshift is determined by the error of the peak wavelength and errors of these two redshifts, which is 
Table 3

A Summary of the Fitting Results for PG QSOs

\begin{tabular}{|c|c|c|c|c|c|c|c|c|}
\hline (1) & Name & (3) & $\begin{array}{c}L_{\mathrm{N}} \\
\left(\mathrm{erg} \mathrm{s}^{-1}\right) \\
(4)\end{array}$ & $\begin{array}{c}\mathrm{FWHM}_{\mathrm{N}} \\
\left(\mathrm{km} \mathrm{s}^{-1}\right) \\
(5)\end{array}$ & $\begin{array}{c}\operatorname{Peak}_{\mathrm{N}} \\
\left(\mathrm{km} \mathrm{s}^{-1}\right) \\
(6)\end{array}$ & $\begin{array}{c}L_{\mathrm{B}} \\
\left(\mathrm{erg} \mathrm{s}^{-1}\right) \\
(7)\end{array}$ & $\begin{array}{c}\mathrm{FWHM}_{\mathrm{b}} \\
\left(\mathrm{km} \mathrm{s}^{-1}\right) \\
(8)\end{array}$ & $\begin{array}{c}\text { Peak }_{\mathrm{B}} \\
\left(\mathrm{km} \mathrm{s}^{-1}\right) \\
(9)\end{array}$ \\
\hline 1 & $0003+158$ & 0.450 & $44.28 \pm 0.01$ & $2558 \pm 58$ & $5 \pm 20$ & $44.67 \pm 0.01$ & $12357 \pm 223$ & $-847 \pm 72$ \\
\hline 2 & $0003+199$ & 0.025 & $42.70 \pm 0.01$ & $2349 \pm 37$ & $-223 \pm 14$ & $42.58 \pm 0.01$ & $14492 \pm 619$ & $192 \pm 188$ \\
\hline 3 & $0007+106$ & 0.089 & $42.97 \pm 0.02$ & $3003 \pm 122$ & $-170 \pm 41$ & $43.17 \pm 0.02$ & $15174 \pm 026$ & $-509 \pm 277$ \\
\hline 4 & $0026+129$ & 0.142 & $42.93 \pm 0.02$ & $1536 \pm 69$ & $-1006 \pm 27$ & $43.50 \pm 0.01$ & $14231 \pm 590$ & $-932 \pm 187$ \\
\hline 5 & $0049+171$ & 0.064 & $42.81 \pm 0.14$ & $4556 \pm 816$ & $-386 \pm 231$ & $42.72 \pm 0.16$ & $10520 \pm 282$ & $113 \pm 501$ \\
\hline 6 & $0050+124$ & 0.061 & $42.61 \pm 0.02$ & $2457 \pm 87$ & $1369 \pm 34$ & $42.80 \pm 0.02$ & $20000 \pm 521$ & $2905 \pm 442$ \\
\hline 7 & $0052+251$ & 0.155 & $43.66 \pm 0.02$ & $4134 \pm 147$ & $823 \pm 43$ & $43.98 \pm 0.01$ & $14621 \pm 475$ & $207 \pm 120$ \\
\hline 8 & $0157+001$ & 0.164 & $43.38 \pm 0.02$ & $3688 \pm 143$ & $1872 \pm 41$ & $43.57 \pm 0.02$ & $20000 \pm 488$ & $2675 \pm 337$ \\
\hline 9 & $0804+761$ & 0.100 & $43.46 \pm 0.02$ & $3467 \pm 152$ & $-36 \pm 52$ & $43.59 \pm 0.02$ & $15703 \pm 126$ & $466 \pm 282$ \\
\hline 10 & $0838+770$ & 0.131 & $42.52 \pm 0.27$ & $2208 \pm 712$ & $-355 \pm 178$ & $43.28 \pm 0.06$ & $7955 \pm 842$ & $-1149 \pm 239$ \\
\hline 11 & $0844+349$ & 0.064 & $42.76 \pm 0.06$ & $3535 \pm 357$ & $-197 \pm 102$ & $43.00 \pm 0.05$ & $20000 \pm 272$ & $2197 \pm 877$ \\
\hline 12 & $0921+525$ & 0.035 & $42.61 \pm 0.02$ & $2900 \pm 98$ & $-491 \pm 30$ & $42.50 \pm 0.02$ & $9123 \pm 409$ & $-744 \pm 115$ \\
\hline 13 & $0923+129$ & 0.190 & $43.80 \pm 0.07$ & $2528 \pm 218$ & $-389 \pm 59$ & $44.05 \pm 0.04$ & $6831 \pm 443$ & $-320 \pm 114$ \\
\hline 14 & $0923+201$ & 0.029 & $41.24 \pm 0.10$ & $2695 \pm 433$ & $-817 \pm 135$ & $41.87 \pm 0.03$ & $9901 \pm 723$ & $-2057 \pm 263$ \\
\hline 15 & $0947+396$ & 0.206 & $43.51 \pm 0.02$ & $3030 \pm 94$ & $267 \pm 30$ & $43.70 \pm 0.01$ & $12678 \pm 454$ & $-141 \pm 132$ \\
\hline 16 & $0953+414$ & 0.239 & $43.81 \pm 0.02$ & $2619 \pm 86$ & $1336 \pm 30$ & $44.07 \pm 0.01$ & $11287 \pm 318$ & $657 \pm 108$ \\
\hline 17 & $1011-040$ & 0.058 & $42.21 \pm 0.04$ & $2516 \pm 228$ & $-4 \pm 81$ & $42.63 \pm 0.04$ & $18485 \pm 930$ & $-65 \pm 747$ \\
\hline 18 & $1012+008$ & 0.185 & $42.42 \pm 0.11$ & $2014 \pm 359$ & $968 \pm 117$ & $43.03 \pm 0.03$ & $7275 \pm 520$ & $-130 \pm 228$ \\
\hline 19 & $1022+519$ & 0.045 & $41.90 \pm 0.08$ & $1991 \pm 324$ & $-31 \pm 115$ & $42.45 \pm 0.04$ & $11474 \pm 412$ & $412 \pm 434$ \\
\hline 20 & $1048-090$ & 0.344 & $43.43 \pm 0.22$ & $3098 \pm 1229$ & $-989 \pm 434$ & $44.13 \pm 0.06$ & $15904 \pm 346$ & $1199 \pm 127$ \\
\hline 21 & $1048+342$ & 0.167 & $42.08 \pm 0.41$ & $1840 \pm 1048$ & $410 \pm 331$ & $42.56 \pm 0.17$ & $5803 \pm 853$ & $1301 \pm 891$ \\
\hline 22 & 1049-005 & 0.357 & $44.09 \pm 0.01$ & $3715 \pm 77$ & $-65 \pm 26$ & $44.20 \pm 0.01$ & $19891 \pm 065$ & $-174 \pm 252$ \\
\hline 23 & $1100+772$ & 0.313 & $43.65 \pm 0.02$ & $2066 \pm 86$ & $352 \pm 34$ & $44.39 \pm 0.01$ & $13162 \pm 199$ & $490 \pm 69$ \\
\hline 24 & $1103-006$ & 0.425 & $43.20 \pm 0.09$ & $2000 \pm 294$ & $-55 \pm 89$ & $43.88 \pm 0.02$ & $7454 \pm 396$ & $-5 \pm 124$ \\
\hline 25 & $1115+407$ & 0.154 & $43.11 \pm 0.04$ & $4013 \pm 251$ & $529 \pm 77$ & $43.26 \pm 0.03$ & $18102 \pm 370$ & $-292 \pm 590$ \\
\hline 26 & $1116+215$ & 0.177 & $43.90 \pm 0.02$ & $3750 \pm 134$ & $925 \pm 41$ & $44.10 \pm 0.01$ & $14092 \pm 599$ & $1032 \pm 154$ \\
\hline 27 & $1119+120$ & 0.049 & $42.22 \pm 0.05$ & $2769 \pm 259$ & $-40 \pm 91$ & $42.73 \pm 0.02$ & $17052 \pm 525$ & $620 \pm 425$ \\
\hline 28 & $1121+422$ & 0.234 & $43.68 \pm 0.01$ & $2297 \pm 60$ & $2580 \pm 23$ & $43.60 \pm 0.02$ & $13480 \pm 798$ & $2168 \pm 265$ \\
\hline 29 & $1149-110$ & 0.049 & $42.38 \pm 0.04$ & $4100 \pm 255$ & $570 \pm 79$ & $42.44 \pm 0.03$ & $15317 \pm 652$ & $1795 \pm 453$ \\
\hline 30 & $1151+117$ & 0.176 & $43.08 \pm 0.04$ & $2406 \pm 190$ & $-23 \pm 66$ & $43.37 \pm 0.03$ & $12044 \pm 165$ & $-1729 \pm 440$ \\
\hline 31 & $1202+281$ & 0.165 & $43.32 \pm 0.01$ & $2565 \pm 72$ & $673 \pm 27$ & $43.45 \pm 0.01$ & $11340 \pm 327$ & $1258 \pm 111$ \\
\hline 32 & $1211+143$ & 0.085 & $43.20 \pm 0.02$ & $2434 \pm 94$ & $-196 \pm 32$ & $43.22 \pm 0.02$ & $10480 \pm 678$ & $419 \pm 229$ \\
\hline 33 & $1216+069$ & 0.334 & $43.96 \pm 0.01$ & $2509 \pm 65$ & $281 \pm 24$ & $44.35 \pm 0.01$ & $15104 \pm 376$ & $864 \pm 115$ \\
\hline 34 & $1226+023$ & 0.158 & $44.11 \pm 0.02$ & $3004 \pm 91$ & $622 \pm 29$ & $44.34 \pm 0.01$ & $10603 \pm 308$ & $-492 \pm 111$ \\
\hline 35 & $1229+204$ & 0.064 & $42.89 \pm 0.03$ & $3632 \pm 161$ & $445 \pm 48$ & $42.91 \pm 0.03$ & $12725 \pm 967$ & $-264 \pm 272$ \\
\hline 36 & $1259+593$ & 0.472 & $43.53 \pm 0.17$ & $4141 \pm 862$ & $1700 \pm 252$ & $44.13 \pm 0.04$ & $11075 \pm 739$ & $2905 \pm 282$ \\
\hline 37 & $1302-102$ & 0.286 & $43.76 \pm 0.02$ & $3234 \pm 138$ & $1905 \pm 48$ & $44.21 \pm 0.01$ & $20000 \pm 076$ & $564 \pm 282$ \\
\hline 38 & $1307+085$ & 0.155 & $43.52 \pm 0.02$ & $3556 \pm 131$ & $-264 \pm 43$ & $43.71 \pm 0.01$ & $15519 \pm 821$ & $-256 \pm 207$ \\
\hline 39 & $1310-108$ & 0.035 & $42.30 \pm 0.04$ & $3193 \pm 187$ & $-229 \pm 57$ & $42.32 \pm 0.04$ & $10706 \pm 959$ & $342 \pm 276$ \\
\hline 40 & $1322+659$ & 0.168 & $43.36 \pm 0.01$ & $2976 \pm 53$ & $549 \pm 18$ & $43.30 \pm 0.01$ & $12769 \pm 456$ & $1263 \pm 138$ \\
\hline 41 & $1341+258$ & 0.087 & $41.79 \pm 0.31$ & $1500 \pm 696$ & $-389 \pm 199$ & $42.73 \pm 0.06$ & $6835 \pm 704$ & $-70 \pm 280$ \\
\hline 42 & $1352+183$ & 0.158 & $43.30 \pm 0.03$ & $3957 \pm 187$ & $2256 \pm 58$ & $43.39 \pm 0.02$ & $16238 \pm 294$ & $1635 \pm 329$ \\
\hline 43 & $1402+261$ & 0.164 & $43.46 \pm 0.03$ & $3895 \pm 219$ & $489 \pm 72$ & $43.70 \pm 0.02$ & $17677 \pm 478$ & $1954 \pm 385$ \\
\hline 44 & $1415+451$ & 0.114 & $43.05 \pm 0.02$ & $3473 \pm 125$ & $509 \pm 40$ & $43.05 \pm 0.02$ & $12530 \pm 738$ & $1219 \pm 207$ \\
\hline 45 & $1416-129$ & 0.129 & $43.29 \pm 0.03$ & $3724 \pm 156$ & $-7 \pm 51$ & $43.54 \pm 0.01$ & $13794 \pm 524$ & $313 \pm 141$ \\
\hline 46 & $1425+267$ & 0.366 & $43.84 \pm 0.15$ & $8094 \pm 1032$ & $1555 \pm 165$ & $43.99 \pm 0.07$ & $20000 \pm 970$ & $174 \pm 022$ \\
\hline 47 & $1426+015$ & 0.086 & $43.01 \pm 0.06$ & $3280 \pm 320$ & $49 \pm 101$ & $43.55 \pm 0.02$ & $14245 \pm 929$ & $-221 \pm 251$ \\
\hline 48 & $1427+480$ & 0.221 & $43.41 \pm 0.02$ & $2374 \pm 74$ & $-59 \pm 25$ & $43.53 \pm 0.01$ & $8412 \pm 264$ & $-799 \pm 95$ \\
\hline 49 & $1435-067$ & 0.129 & $43.10 \pm 0.05$ & $2862 \pm 225$ & $227 \pm 66$ & $43.20 \pm 0.04$ & $9868 \pm 053$ & $361 \pm 296$ \\
\hline 50 & $1440+356$ & 0.077 & $42.76 \pm 0.04$ & $1670 \pm 108$ & $468 \pm 31$ & $42.95 \pm 0.03$ & $5346 \pm 350$ & $832 \pm 106$ \\
\hline 51 & $1444+407$ & 0.267 & $43.04 \pm 0.15$ & $2179 \pm 507$ & $748 \pm 159$ & $43.81 \pm 0.03$ & $7761 \pm 508$ & $1440 \pm 173$ \\
\hline 52 & $1501+106$ & 0.036 & $42.50 \pm 0.03$ & $3389 \pm 160$ & $141 \pm 52$ & $42.80 \pm 0.01$ & $13579 \pm 586$ & $617 \pm 162$ \\
\hline 53 & $1512+370$ & 0.371 & $43.79 \pm 0.02$ & $2595 \pm 117$ & $68 \pm 41$ & $44.38 \pm 0.01$ & $12778 \pm 287$ & $1001 \pm 96$ \\
\hline 54 & $1519+226$ & 0.137 & $42.96 \pm 0.04$ & $2432 \pm 188$ & $754 \pm 68$ & $43.23 \pm 0.03$ & $14662 \pm 466$ & $-218 \pm 457$ \\
\hline 55 & $1534+580$ & 0.030 & $42.02 \pm 0.03$ & $1844 \pm 114$ & $-289 \pm 40$ & $42.59 \pm 0.01$ & $8679 \pm 247$ & $323 \pm 87$ \\
\hline 56 & $1543+489$ & 0.400 & $43.80 \pm 0.03$ & $4802 \pm 200$ & $1997 \pm 55$ & $43.81 \pm 0.03$ & $15044 \pm 147$ & $2905 \pm 281$ \\
\hline 57 & $1545+210$ & 0.266 & $43.84 \pm 0.03$ & $3830 \pm 177$ & $230 \pm 58$ & $44.21 \pm 0.01$ & $15845 \pm 617$ & $302 \pm 160$ \\
\hline 58 & $1612+261$ & 0.131 & $43.06 \pm 0.03$ & $2781 \pm 137$ & $-285 \pm 47$ & $43.38 \pm 0.01$ & $10543 \pm 379$ & $-1724 \pm 153$ \\
\hline 59 & $1613+658$ & 0.129 & $43.41 \pm 0.04$ & $5136 \pm 273$ & $574 \pm 74$ & $43.53 \pm 0.03$ & $14,978 \pm 225$ & $-1793 \pm 502$ \\
\hline 60 & $1617+175$ & 0.114 & $42.50 \pm 0.10$ & $2810 \pm 458$ & $354 \pm 146$ & $43.10 \pm 0.03$ & $12214 \pm 049$ & $415 \pm 308$ \\
\hline 61 & $1626+554$ & 0.133 & $43.25 \pm 0.03$ & $3366 \pm 168$ & $569 \pm 54$ & $43.45 \pm 0.02$ & $13619 \pm 804$ & $-74 \pm 239$ \\
\hline 62 & $2130+099$ & 0.061 & $42.74 \pm 0.03$ & $2226 \pm 118$ & $-518 \pm 40$ & $42.94 \pm 0.02$ & $10361 \pm 670$ & $-337 \pm 211$ \\
\hline
\end{tabular}


Table 3

(Continued)

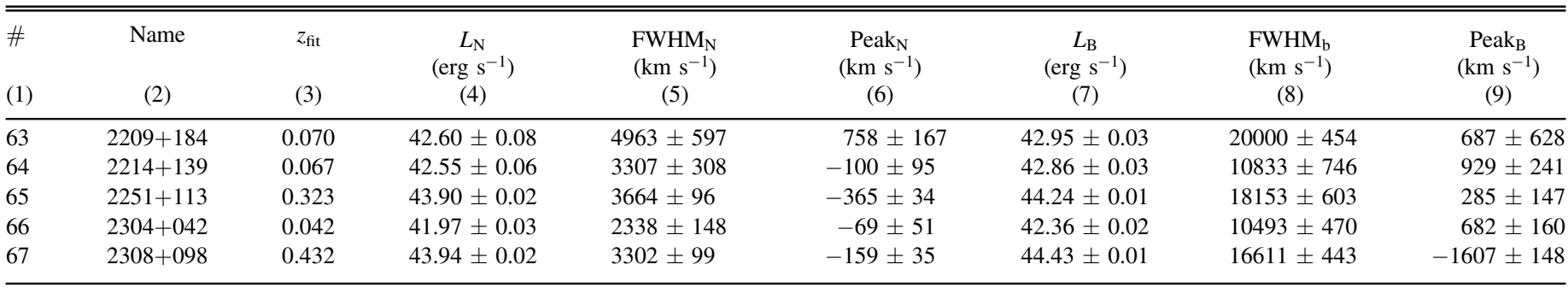

Note. Column (1): sequence number; column (2): name of PG QSOs; column (3): redshift from Schmidt \& Green (1983); columns (4)-(9): the profile fitting parameters of C IV (luminosity, FWHM, and peak). Subscript N and B indicate narrow and broad components, respectively.

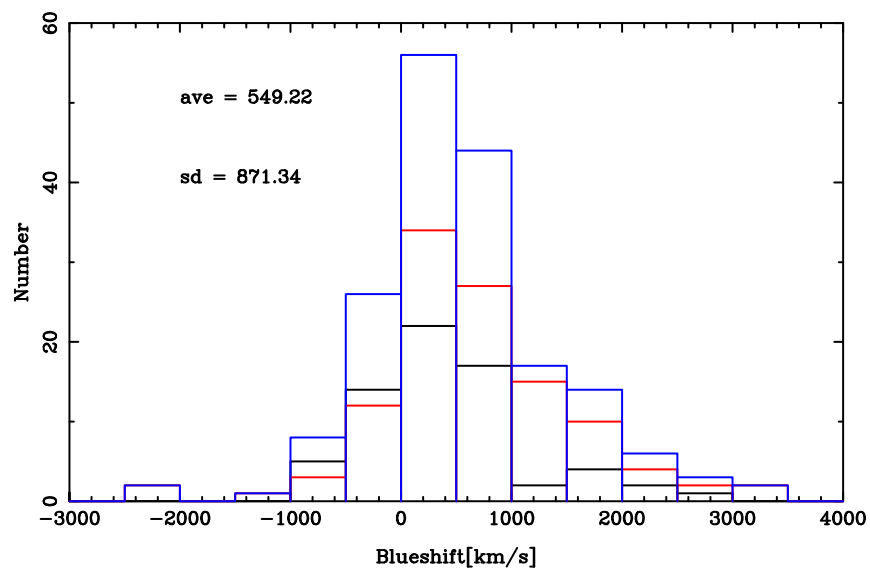

Figure 3. Distributions of the velocity shift of the whole C IV emission line for PG QSOs (black), high-z QSOs (red), and the entire sample (blue). The average and standard deviation of the distribution are marked on the top right. The positive value denotes the blueshift in units of $\mathrm{km} \mathrm{s}^{-1}$.

related to the spectral resolution during observations.

$$
\begin{gathered}
v=\left(\frac{\lambda\left(1+z_{\mathrm{fit}}\right)}{1+z_{\mathrm{sys}}}-1549\right) \frac{c}{1549} \\
(\delta v)^{2}=\left(\left(\frac{\delta \lambda\left(1+z_{\mathrm{fit}}\right)}{1+z_{\mathrm{sys}}}\right)^{2}+\left(\frac{\lambda \delta z_{\mathrm{fit}}}{1+z_{\mathrm{sys}}}\right)^{2}\right. \\
\left.+\left(\frac{\lambda \delta z_{\mathrm{sys}}\left(1+z_{\mathrm{fit}}\right)}{\left(1+z_{\mathrm{sys}}\right)^{2}}\right)^{2}\right) \times\left(\frac{c}{1549}\right)^{2} .
\end{gathered}
$$

We calculate the error of the C IV blueshift according to the error transfer formula (Equation (2)). For PG QSOs, the resolution of the optical spectra is $6.5 \AA$ corresponding to $\sim 400 \mathrm{~km} \mathrm{~s}^{-1}$. The resolution of the UV spectra is $\sim 460 \mathrm{~km} \mathrm{~s}^{-1}$ and $\sim 1200 \mathrm{~km} \mathrm{~s}^{-1}$ for $H S T$ and IUE data, respectively. For a high- $z$ QSO $(z=2)$ with an infrared spectral resolution of $R \sim 100$, the uncertainty of its blueshift is $\sim 1000 \mathrm{~km} \mathrm{~s}^{-1}$. If $R \sim 3000$, then the uncertainty of its blueshift is $\sim 30 \mathrm{~km} \mathrm{~s}^{-1}$. For SDSS UV spectra, $R \sim 2000$, and the uncertainty of the blueshifts is $\sim 50 \mathrm{~km} \mathrm{~s}^{-1}$. Typically, the centroid of a wellshaped emission line can be determined to at least 0.1 or better of the spectral resolution. The larger uncertainty in the C IV blueshift comes from noise in the broad emission line profiles (Boroson \& Green 1992). We can therefore ignore the effect of spectral resolution on the error of the blueshift. For that reason, we do not consider the uncertainties from the spectral

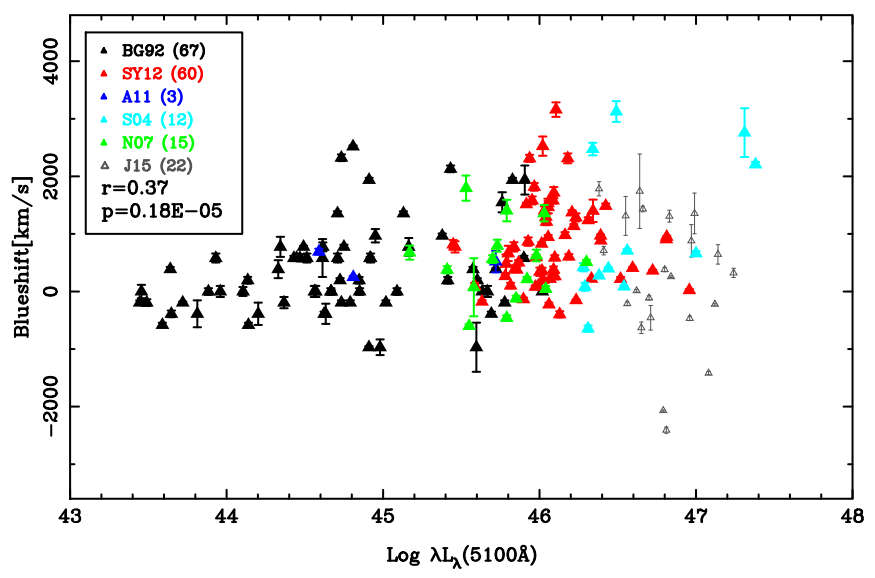

Figure 4. C IV velocity shift vs. the luminosity at $5100 \AA$ A for our sample. The points with different colors represent that these sources are form different literatures and the number in the brackets indicates the number of objects used here as indicated in the legend. A subsample without the sources from Jun et al. (2015; gray points) is used to do the Spearman correlation test. The Spearman correlation coefficient and the probability of the null hypothesis are 0.37 and $1.8 \times 10^{-6}$, respectively.

resolution and the two redshifts, zfit and $z_{\text {sys }}$, for either lowor high- $z$ QSOs. The error of the C IV blueshift is calculated only from the error of the fit of the C IV peak wavelength.

For our sample of low- $z$ and high- $z$ QSOs, Ge et al. (2016) has computed the $\mathrm{H} \beta$-based single-epoch SMBH mass, $M_{\mathrm{BH}}$, and host-corrected $L_{\mathrm{Bol}} / L_{\mathrm{Edd}}$. They estimated the bolometric luminosity based on a constant bolometric correction (9.26) and host-corrected $L(5100 \AA$; see Ge et al. 2016 for details) . We use these parameters to investigate the relation with the C IV blueshift. In order to investigate the bias in C IV-based $M_{\mathrm{BH}}$, we measure the C IV FWHM from the best-fitting C IV profile, as well as the luminosity at $1350 \AA$. The C IV-based SMBH mass is calculated following the formula of Vestergaard $\&$ Peterson (2006).

\section{Result and Discussion}

\subsection{Blueshift of the Whole CIV Emission Line}

Based on the rest frame defined by [O III] $\lambda 5007$ or other lowionization emission lines (e.g., $\mathrm{H} \beta$ ), the blueshift of the whole $\mathrm{C}$ IV profile is investigated for our sample. Figure 3 shows the distribution of the C IV velocity shift for low-z PG QSOs (black), high- $z$ QSOs (red), and our sample (blue). Most objects exhibit blueshift (positive values) and the biggest blueshift is up to $\sim 3200 \mathrm{~km} \mathrm{~s}^{-1}$. The average and standard deviation of the 
Table 4

A Summary of the Fitting Results for High- $z$ QSOs

\begin{tabular}{|c|c|c|c|c|c|c|c|c|}
\hline (1) & Name & (3) & $\begin{array}{c}L_{\mathrm{N}} \\
\left(\mathrm{erg} \mathrm{s}^{-1}\right) \\
(4)\end{array}$ & $\begin{array}{c}\mathrm{FWHM}_{\mathrm{N}} \\
\left(\mathrm{km} \mathrm{s}^{-1}\right) \\
(5)\end{array}$ & $\begin{array}{c}\text { Peak }_{\mathrm{N}} \\
\left(\mathrm{km} \mathrm{s}^{-1}\right) \\
(6)\end{array}$ & $\begin{array}{c}L_{\mathrm{B}} \\
\left(\mathrm{erg} \mathrm{s}^{-1}\right) \\
(7)\end{array}$ & $\begin{array}{c}\mathrm{FWHM}_{\mathrm{b}} \\
\left(\mathrm{km} \mathrm{s}^{-1}\right) \\
(8)\end{array}$ & $\begin{array}{c}\text { Peak }_{\mathrm{B}} \\
\left(\mathrm{km} \mathrm{s}^{-1}\right) \\
(9)\end{array}$ \\
\hline 1 & SDSS225800.02-084143 & 1.496 & $45.54 \pm 0.05$ & $3753 \pm 226$ & $807 \pm 80$ & $46.00 \pm 0.07$ & $16574 \pm 1581$ & $2905 \pm 131$ \\
\hline 2 & SDSS035856.73-054023 & 1.506 & $45.04 \pm 0.11$ & $3454 \pm 446$ & $1007 \pm 133$ & $45.24 \pm 0.08$ & $8050 \pm 676$ & $-1079 \pm 614$ \\
\hline 3 & SDSS133321.90+005824 & 1.511 & $45.64 \pm 0.02$ & $2794 \pm 86$ & $361 \pm 30$ & $46.25 \pm 0.01$ & $12624 \pm 247$ & $-910 \pm 80$ \\
\hline 4 & SDSS081331.28+254503 & 1.513 & $46.42 \pm 0.02$ & $3394 \pm 83$ & $843 \pm 19$ & $46.48 \pm 0.01$ & $10223 \pm 406$ & $1349 \pm 92$ \\
\hline 5 & SDSS152111.86+470539 & 1.517 & $45.73 \pm 0.02$ & $2664 \pm 74$ & $409 \pm 25$ & $45.98 \pm 0.01$ & $12977 \pm 565$ & $21 \pm 163$ \\
\hline 6 & FBQ1633+3134 & 1.522 & $45.32 \pm 0.12$ & $2265 \pm 256$ & $2905 \pm 185$ & $45.69 \pm 0.05$ & $3073 \pm 239$ & $571 \pm 175$ \\
\hline 7 & SDSS085543.26+002908 & 1.525 & $45.43 \pm 0.02$ & $2664 \pm 93$ & $149 \pm 30$ & $45.82 \pm 0.01$ & $12712 \pm 494$ & $770 \pm 143$ \\
\hline 8 & SDSS123355.21+031327 & 1.528 & $45.41 \pm 0.05$ & $4380 \pm 261$ & $954 \pm 71$ & $45.99 \pm 0.01$ & $14673 \pm 693$ & $1088 \pm 164$ \\
\hline 9 & SDSS104910.31+143227 & 1.540 & $45.74 \pm 0.02$ & $4331 \pm 112$ & $1265 \pm 29$ & $45.67 \pm 0.02$ & $12566 \pm 750$ & $1082 \pm 160$ \\
\hline 10 & SDSS154212.90+111226 & 1.540 & $45.93 \pm 0.14$ & $5052 \pm 521$ & $1315 \pm 106$ & $45.43 \pm 0.47$ & $9174 \pm 5240$ & $-2905 \pm 310$ \\
\hline 11 & SDSS143230.57+012435 & 1.542 & $45.64 \pm 0.04$ & $4443 \pm 248$ & $1454 \pm 67$ & $45.82 \pm 0.03$ & $17032 \pm 2317$ & $2095 \pm 516$ \\
\hline 12 & SDSS074029.82+281458 & 1.545 & $45.82 \pm 0.03$ & $3925 \pm 136$ & $970 \pm 36$ & $46.09 \pm 0.01$ & $13315 \pm 662$ & $2198 \pm 163$ \\
\hline 13 & SDSS081344.15+152221 & 1.545 & $45.62 \pm 0.07$ & $4693 \pm 351$ & $1349 \pm 75$ & $45.72 \pm 0.04$ & $13894 \pm 2885$ & $2905 \pm 962$ \\
\hline 14 & SDSS082146.22+571226 & 1.546 & $45.99 \pm 0.02$ & $4771 \pm 167$ & $1596 \pm 49$ & $45.74 \pm 0.08$ & $20000 \pm 5902$ & $2905 \pm 753$ \\
\hline 15 & SDSS171030.20+602347 & 1.549 & $45.69 \pm 0.03$ & $3888 \pm 172$ & $566 \pm 54$ & $45.98 \pm 0.02$ & $11419 \pm 400$ & $-1210 \pm 177$ \\
\hline 16 & SDSS123442.16+052126 & 1.550 & $45.73 \pm 0.03$ & $4507 \pm 188$ & $1091 \pm 52$ & $45.86 \pm 0.02$ & $13,433 \pm 901$ & $267 \pm 213$ \\
\hline 17 & SDSS015733.87-004824 & 1.551 & $45.56 \pm 0.09$ & $6586 \pm 562$ & $1578 \pm 162$ & $45.27 \pm 0.17$ & $13578 \pm 2649$ & $-2905 \pm 531$ \\
\hline 18 & SDSS101447.54+521320 & 1.552 & $45.71 \pm 0.04$ & $4261 \pm 210$ & $689 \pm 54$ & $45.59 \pm 0.04$ & $13448 \pm 1971$ & $458 \pm 395$ \\
\hline 19 & SDSS135439.70+301649 & 1.553 & $45.69 \pm 0.02$ & $4852 \pm 155$ & $1182 \pm 45$ & $45.54 \pm 0.04$ & $19307 \pm 3631$ & $-406 \pm 755$ \\
\hline 20 & SDSS223246.80+134702 & 1.557 & $45.49 \pm 0.02$ & $2659 \pm 106$ & $155 \pm 34$ & $46.14 \pm 0.01$ & $10761 \pm 182$ & $-27 \pm 54$ \\
\hline 21 & SDSS100930.51+023052 & 1.557 & $45.47 \pm 0.03$ & $4986 \pm 239$ & $343 \pm 52$ & $45.65 \pm 0.02$ & $20000 \pm 2813$ & $-75 \pm 359$ \\
\hline 22 & SDSS124006.70+474003 & 1.561 & $45.68 \pm 0.03$ & $3652 \pm 146$ & $495 \pm 40$ & $45.94 \pm 0.02$ & $9284 \pm 296$ & $1512 \pm 96$ \\
\hline 23 & SDSS093318.49+141340 & 1.561 & $45.86 \pm 0.02$ & $4378 \pm 128$ & $834 \pm 53$ & $45.96 \pm 0.02$ & $12342 \pm 402$ & $-1432 \pm 192$ \\
\hline 24 & SDSS094126.49+044328 & 1.567 & $45.54 \pm 0.03$ & $3350 \pm 146$ & $284 \pm 44$ & $45.97 \pm 0.01$ & $11759 \pm 375$ & $-509 \pm 108$ \\
\hline 25 & SDSS113829.33+040101 & 1.567 & $45.06 \pm 0.05$ & $1406 \pm 151$ & $474 \pm 55$ & $46.11 \pm 0.01$ & $8871 \pm 198$ & $1281 \pm 72$ \\
\hline 26 & SDSS084451.91+282607 & 1.574 & $45.83 \pm 0.02$ & $4686 \pm 105$ & $1307 \pm 29$ & $45.69 \pm 0.02$ & $20000 \pm 2320$ & $-60 \pm 506$ \\
\hline 27 & SDSS081227.19+075732 & 1.574 & $45.37 \pm 0.05$ & $3321 \pm 229$ & $315 \pm 72$ & $45.80 \pm 0.02$ & $9313 \pm 298$ & $-1703 \pm 174$ \\
\hline 28 & SDSS091754.44+043652 & 1.587 & $45.74 \pm 0.02$ & $2862 \pm 94$ & $248 \pm 31$ & $46.22 \pm 0.01$ & $9575 \pm 150$ & $-954 \pm 59$ \\
\hline 29 & SDSS204538.96-005115 & 1.590 & $45.48 \pm 0.04$ & $3077 \pm 178$ & $844 \pm 56$ & $45.84 \pm 0.02$ & $12037 \pm 640$ & $244 \pm 179$ \\
\hline 30 & SDSS014705.42+133210 & 1.595 & $45.64 \pm 0.03$ & $3659 \pm 139$ & $969 \pm 40$ & $46.02 \pm 0.01$ & $12681 \pm 386$ & $1740 \pm 107$ \\
\hline 31 & SDSS114023.40+301651 & 1.599 & $46.01 \pm 0.03$ & $4511 \pm 161$ & $1775 \pm 43$ & $46.31 \pm 0.01$ & $13585 \pm 424$ & $2205 \pm 100$ \\
\hline 32 & SDSS104603.22+112828 & 1.607 & $45.61 \pm 0.03$ & $3861 \pm 156$ & $991 \pm 44$ & $45.78 \pm 0.02$ & $12578 \pm 607$ & $422 \pm 168$ \\
\hline 33 & SDSS125140.82+080718 & 1.607 & $45.53 \pm 0.08$ & $3991 \pm 359$ & $1751 \pm 97$ & $46.14 \pm 0.02$ & $9438 \pm 278$ & $2905 \pm 103$ \\
\hline 34 & SDSS204009.62-065402 & 1.611 & $45.09 \pm 0.07$ & $2830 \pm 328$ & $647 \pm 97$ & $45.45 \pm 0.03$ & $10093 \pm 860$ & $433 \pm 246$ \\
\hline 35 & SDSS155240.40+194816 & 1.613 & $45.65 \pm 0.04$ & $4452 \pm 213$ & $1172 \pm 60$ & $45.86 \pm 0.02$ & $13716 \pm 776$ & $-544 \pm 270$ \\
\hline 36 & SDSS002948.04-095639 & 1.618 & $45.90 \pm 0.01$ & $3728 \pm 81$ & $802 \pm 24$ & $45.92 \pm 0.01$ & $13304 \pm 527$ & $-36 \pm 150$ \\
\hline 37 & SDSS083850.15+261105 & 1.618 & $46.34 \pm 0.01$ & $3959 \pm 57$ & $987 \pm 19$ & $46.45 \pm 0.01$ & $16928 \pm 446$ & $1930 \pm 112$ \\
\hline 38 & SDSS205554.08+004311 & 1.624 & $45.45 \pm 0.05$ & $4117 \pm 274$ & $1020 \pm 78$ & $45.54 \pm 0.04$ & $15466 \pm 2008$ & $1493 \pm 469$ \\
\hline 39 & SDSS135023.68+265243 & 1.624 & $45.69 \pm 0.02$ & $3036 \pm 108$ & $834 \pm 36$ & $46.11 \pm 0.01$ & $12265 \pm 307$ & $2041 \pm 105$ \\
\hline 40 & SDSS111949.30+233249 & 1.626 & $45.39 \pm 0.02$ & $1548 \pm 61$ & $417 \pm 21$ & $45.92 \pm 0.01$ & $6682 \pm 207$ & $428 \pm 65$ \\
\hline 41 & SDSS004149.64-094705 & 1.629 & $45.88 \pm 0.02$ & $4764 \pm 152$ & $650 \pm 46$ & $46.30 \pm 0.01$ & $20000 \pm 777$ & $2140 \pm 168$ \\
\hline 42 & SDSS160456.14-001907 & 1.636 & $45.70 \pm 0.04$ & $4019 \pm 243$ & $1377 \pm 70$ & $46.16 \pm 0.02$ & $12523 \pm 462$ & $2422 \pm 144$ \\
\hline 43 & SDSS141949.39+060654 & 1.649 & $45.86 \pm 0.04$ & $5696 \pm 251$ & $2020 \pm 62$ & $45.88 \pm 0.03$ & $18045 \pm 1835$ & $2905 \pm 346$ \\
\hline 44 & SDSS020044.50+122319 & 1.654 & $45.65 \pm 0.02$ & $3101 \pm 116$ & $719 \pm 40$ & $45.94 \pm 0.01$ & $12268 \pm 448$ & $-1676 \pm 203$ \\
\hline 45 & SDSS142841.97+592552 & 1.660 & $45.47 \pm 0.04$ & $3085 \pm 190$ & $1031 \pm 53$ & $45.96 \pm 0.01$ & $9632 \pm 313$ & $1536 \pm 90$ \\
\hline 46 & SDSS204536.56-010147 & 1.661 & $46.37 \pm 0.02$ & $4861 \pm 119$ & $981 \pm 35$ & $46.34 \pm 0.02$ & $13112 \pm 515$ & $-1416 \pm 246$ \\
\hline 47 & SDSS110240.16+394730 & 1.664 & $45.78 \pm 0.04$ & $5992 \pm 288$ & $1948 \pm 71$ & $45.72 \pm 0.03$ & $18326 \pm 2498$ & $-423 \pm 821$ \\
\hline 48 & SDSS100401.27+423123 & 1.666 & $45.55 \pm 0.19$ & $4071 \pm 642$ & $1570 \pm 194$ & $46.14 \pm 0.05$ & $7435 \pm 282$ & $2859 \pm 179$ \\
\hline 49 & SDSS213748.44+001220 & 1.670 & $45.36 \pm 0.03$ & $2630 \pm 138$ & $659 \pm 47$ & $44.58 \pm 0.26$ & $11711 \pm 10655$ & $2905 \pm 887$ \\
\hline 50 & SDSS094913.05+175155 & 1.675 & $45.59 \pm 0.04$ & $4182 \pm 295$ & $1877 \pm 85$ & $46.18 \pm 0.01$ & $15051 \pm 484$ & $2705 \pm 125$ \\
\hline 51 & SDSS153859.45+053705 & 1.684 & $45.76 \pm 0.02$ & $3515 \pm 112$ & $1066 \pm 34$ & $45.81 \pm 0.02$ & $12147 \pm 692$ & $940 \pm 176$ \\
\hline 52 & SDSS162103.98+002905 & 1.689 & $45.20 \pm 0.15$ & $4544 \pm 619$ & $1493 \pm 166$ & $45.49 \pm 0.08$ & $9407 \pm 821$ & $2765 \pm 348$ \\
\hline 53 & SDSS0246-0825 & 1.690 & $45.40 \pm 0.05$ & $2614 \pm 177$ & $547 \pm 51$ & $46.00 \pm 0.01$ & $7018 \pm 166$ & $1428 \pm 63$ \\
\hline 54 & SDSS105951.05+090905 & 1.690 & $46.35 \pm 0.01$ & $2421 \pm 28$ & $521 \pm 10$ & $46.47 \pm 0.01$ & $11605 \pm 172$ & $647 \pm 54$ \\
\hline 55 & SDSS041255.16-061210 & 1.691 & $45.75 \pm 0.06$ & $4507 \pm 264$ & $1159 \pm 77$ & $46.07 \pm 0.03$ & $10093 \pm 351$ & $2780 \pm 164$ \\
\hline 56 & SDSS112542.29+000101 & 1.692 & $46.25 \pm 0.01$ & $2172 \pm 24$ & $301 \pm 12$ & $46.10 \pm 0.01$ & $8868 \pm 187$ & $-1635 \pm 121$ \\
\hline 57 & SDSS101504.75+123022 & 1.703 & $45.68 \pm 0.10$ & $5866 \pm 591$ & $2540 \pm 125$ & $46.04 \pm 0.04$ & $15706 \pm 1470$ & $2905 \pm 241$ \\
\hline 58 & SDSS122039.45+000427 & 2.048 & $46.29 \pm 0.02$ & $4344 \pm 104$ & $1058 \pm 29$ & $46.54 \pm 0.01$ & $14361 \pm 377$ & $890 \pm 89$ \\
\hline 59 & SDSS143645.80+633637 & 2.066 & $46.38 \pm 0.01$ & $2989 \pm 54$ & $336 \pm 17$ & $46.73 \pm 0.01$ & $11201 \pm 146$ & $355 \pm 42$ \\
\hline 60 & SDSS014944.43+150106 & 2.073 & $46.52 \pm 0.01$ & $5495 \pm 89$ & $1488 \pm 22$ & $46.51 \pm 0.01$ & $18675 \pm 891$ & $656 \pm 188$ \\
\hline 61 & SDSS143148.09+053558 & 2.095 & $46.54 \pm 0.01$ & $3676 \pm 73$ & $445 \pm 22$ & $46.82 \pm 0.01$ & $14190 \pm 293$ & $348 \pm 77$ \\
\hline 62 & SDSS142108.71+224117 & 2.188 & $46.88 \pm 0.01$ & $4322 \pm 51$ & $655 \pm 17$ & $46.83 \pm 0.01$ & $14387 \pm 329$ & $944 \pm 78$ \\
\hline
\end{tabular}


Table 4

(Continued)

\begin{tabular}{|c|c|c|c|c|c|c|c|c|}
\hline (1) & $\begin{array}{c}\text { Name } \\
(2)\end{array}$ & (3) & $\begin{array}{c}L_{\mathrm{N}} \\
\left(\operatorname{erg~s}^{-1}\right) \\
(4)\end{array}$ & $\begin{array}{c}\mathrm{FWHM}_{\mathrm{N}} \\
\left(\mathrm{km} \mathrm{s}^{-1}\right) \\
(5)\end{array}$ & $\begin{array}{c}\operatorname{Peak}_{\mathrm{N}} \\
\left(\mathrm{km} \mathrm{s}^{-1}\right) \\
(6)\end{array}$ & $\begin{array}{c}L_{\mathrm{B}} \\
\left(\operatorname{erg~s}^{-1}\right) \\
(7)\end{array}$ & $\begin{array}{c}\mathrm{FWHM}_{\mathrm{b}} \\
\left(\mathrm{km} \mathrm{s}^{-1}\right) \\
(8)\end{array}$ & $\begin{array}{c}\text { Peak }_{\mathrm{B}} \\
\left(\mathrm{km} \mathrm{s}^{-1}\right) \\
(9)\end{array}$ \\
\hline 63 & TON618 & 2.219 & $45.44 \pm 0.35$ & $3083 \pm 1398$ & $1331 \pm 423$ & $46.66 \pm 0.02$ & $8620 \pm 362$ & $2905 \pm 179$ \\
\hline 64 & UM645 & 2.268 & $46.35 \pm 0.03$ & $4677 \pm 202$ & $360 \pm 54$ & $46.35 \pm 0.02$ & $18706 \pm 2100$ & $172 \pm 480$ \\
\hline 65 & SDSSJ170102.18+61230 & 2.290 & $45.99 \pm 0.05$ & $4196 \pm 286$ & $1437 \pm 110$ & $46.03 \pm 0.05$ & $13274 \pm 1328$ & $2905 \pm 466$ \\
\hline 66 & SDSSJ115111.20+03404 & 2.337 & $45.21 \pm 0.68$ & $1847 \pm 602$ & $292 \pm 504$ & $45.18 \pm 0.75$ & $2483 \pm 1817$ & $-1139 \pm 825$ \\
\hline 67 & SDSSJ144245.66-02425 & 2.355 & $45.80 \pm 0.11$ & $4455 \pm 585$ & $1322 \pm 144$ & $45.79 \pm 0.12$ & $10632 \pm 1897$ & $369 \pm 595$ \\
\hline 68 & SDSSJ100710.70+04211 & 2.367 & $45.79 \pm 0.07$ & $4727 \pm 448$ & $1054 \pm 121$ & $45.64 \pm 0.09$ & $14292 \pm 3832$ & $-1102 \pm 471$ \\
\hline 69 & UM642 & 2.372 & $46.34 \pm 0.03$ & $3865 \pm 190$ & $1042 \pm 69$ & $46.63 \pm 0.01$ & $14602 \pm 680$ & $224 \pm 187$ \\
\hline 70 & SDSSJ125034.41-01051 & 2.399 & $45.79 \pm 0.04$ & $2905 \pm 179$ & $569 \pm 62$ & $46.08 \pm 0.02$ & $9848 \pm 501$ & $-610 \pm 187$ \\
\hline 71 & SDSSJ095141.33+01325 & 2.419 & $45.52 \pm 0.03$ & $1693 \pm 95$ & $159 \pm 33$ & $46.11 \pm 0.01$ & $8025 \pm 226$ & $-727 \pm 86$ \\
\hline 72 & SDSSJ101257.52+02593 & 2.441 & $45.88 \pm 0.06$ & $4782 \pm 371$ & $1374 \pm 103$ & $45.91 \pm 0.05$ & $14478 \pm 1968$ & $2620 \pm 567$ \\
\hline 73 & SDSS1138+0314 & 2.445 & $46.42 \pm 0.01$ & $2195 \pm 36$ & $517 \pm 20$ & $46.43 \pm 0.01$ & $15787 \pm 474$ & $917 \pm 146$ \\
\hline 74 & SDSSJ025438.37+00213 & 2.464 & $45.92 \pm 0.03$ & $2525 \pm 152$ & $544 \pm 49$ & $46.06 \pm 0.02$ & $11724 \pm 803$ & $888 \pm 250$ \\
\hline 75 & UM629 & 2.471 & $46.55 \pm 0.02$ & $4062 \pm 100$ & $1604 \pm 30$ & $46.64 \pm 0.01$ & $14900 \pm 678$ & $2905 \pm 201$ \\
\hline 76 & SDSSJ024933.42-08345 & 2.494 & $46.42 \pm 0.01$ & $2576 \pm 61$ & $520 \pm 20$ & $46.65 \pm 0.01$ & $9979 \pm 266$ & $1760 \pm 117$ \\
\hline 77 & SDSSJ135445.66+00205 & 2.514 & $46.08 \pm 0.12$ & $5431 \pm 647$ & $1326 \pm 179$ & $46.46 \pm 0.05$ & $11463 \pm 685$ & $2905 \pm 309$ \\
\hline 78 & UM632 & 2.521 & $46.29 \pm 0.01$ & $1891 \pm 55$ & $409 \pm 20$ & $46.84 \pm 0.01$ & $11871 \pm 280$ & $382 \pm 87$ \\
\hline 79 & SDSSJ100428.43+00182 & 3.054 & $46.68 \pm 0.02$ & $2543 \pm 76$ & $925 \pm 25$ & $46.89 \pm 0.01$ & $8744 \pm 243$ & $1983 \pm 88$ \\
\hline 80 & UM667 & 3.122 & $46.36 \pm 0.04$ & $4282 \pm 224$ & $-455 \pm 71$ & $46.72 \pm 0.02$ & $13838 \pm 562$ & $1597 \pm 212$ \\
\hline 81 & SBS1425+606 & 3.197 & $47.50 \pm 0.02$ & $5556 \pm 166$ & $1744 \pm 44$ & $47.80 \pm 0.01$ & $15477 \pm 360$ & $2535 \pm 77$ \\
\hline 82 & SDSSJ083700.82+35055 & 3.316 & $47.00 \pm 0.01$ & $3506 \pm 69$ & $1084 \pm 22$ & $47.03 \pm 0.01$ & $13875 \pm 482$ & $2429 \pm 153$ \\
\hline 83 & SDSSJ210258.21+00202 & 3.342 & $45.73 \pm 0.05$ & $1000 \pm 111$ & $544 \pm 42$ & $46.36 \pm 0.03$ & $9442 \pm 777$ & $467 \pm 281$ \\
\hline 84 & SDSSJ210311.69-06005 & 3.344 & $46.75 \pm 0.02$ & $4099 \pm 151$ & $1037 \pm 38$ & $46.87 \pm 0.02$ & $15152 \pm 789$ & $685 \pm 200$ \\
\hline 85 & SDSSJ113838.26-02060 & 3.347 & $45.98 \pm 0.27$ & $3823 \pm 923$ & $1088 \pm 187$ & $46.36 \pm 0.11$ & $8514 \pm 1396$ & $853 \pm 291$ \\
\hline 86 & SDSSJ083630.55+06204 & 3.384 & $46.16 \pm 0.12$ & $4962 \pm 779$ & $965 \pm 219$ & $46.25 \pm 0.09$ & $17208 \pm 6637$ & $-2905 \pm 248$ \\
\hline 87 & SDSSJ105511.99+02075 & 3.404 & $46.58 \pm 0.04$ & $4317 \pm 237$ & $1423 \pm 67$ & $46.77 \pm 0.02$ & $16550 \pm 1517$ & $426 \pm 384$ \\
\hline 88 & SDSSJ173352.22+54003 & 3.435 & $46.99 \pm 0.02$ & $3271 \pm 116$ & $995 \pm 39$ & $47.24 \pm 0.01$ & $10278 \pm 281$ & $2905 \pm 142$ \\
\hline 89 & SDSSJ115304.62+03595 & 3.437 & $46.19 \pm 0.03$ & $1763 \pm 119$ & $786 \pm 49$ & $46.35 \pm 0.04$ & $13901 \pm 1702$ & $2454 \pm 652$ \\
\hline 90 & SDSSJ115935.64+04242 & 3.456 & $46.25 \pm 0.03$ & $1662 \pm 106$ & $527 \pm 39$ & $46.95 \pm 0.01$ & $10541 \pm 295$ & $-112 \pm 107$ \\
\hline 91 & SDSSJ153725.36-01465 & 3.467 & $46.54 \pm 0.04$ & $4532 \pm 326$ & $1567 \pm 101$ & $46.79 \pm 0.03$ & $20000 \pm 2528$ & $2905 \pm 583$ \\
\hline 92 & SDSSJ164248.71+24030 & 3.497 & $46.85 \pm 0.03$ & $5217 \pm 255$ & $1754 \pm 75$ & $47.11 \pm 0.02$ & $20000 \pm 1567$ & $2905 \pm 317$ \\
\hline 93 & SDSSJ150620.48+46064 & 3.503 & $46.79 \pm 0.06$ & $5562 \pm 401$ & $408 \pm 124$ & $46.97 \pm 0.04$ & $14111 \pm 907$ & $2905 \pm 444$ \\
\hline 94 & SDSSJ075303.33+42313 & 3.595 & $47.00 \pm 0.01$ & $2195 \pm 35$ & $212 \pm 12$ & $47.28 \pm 0.01$ & $10746 \pm 199$ & $512 \pm 61$ \\
\hline 95 & SDSSJ144144.76+47200 & 3.643 & $46.86 \pm 0.02$ & $2604 \pm 95$ & $1270 \pm 31$ & $47.17 \pm 0.01$ & $12317 \pm 485$ & $1713 \pm 165$ \\
\hline 96 & SDSSJ145408.95+51144 & 3.648 & $47.28 \pm 0.02$ & $3716 \pm 96$ & $1009 \pm 29$ & $47.46 \pm 0.01$ & $11995 \pm 323$ & $2043 \pm 113$ \\
\hline 97 & SDSSJ101336.37+56153 & 3.652 & $46.46 \pm 0.17$ & $3482 \pm 872$ & $2144 \pm 353$ & $47.11 \pm 0.03$ & $10524 \pm 586$ & $1721 \pm 154$ \\
\hline 98 & SDSSJ014049.18-08394 & 3.726 & $47.14 \pm 0.02$ & $3867 \pm 124$ & $1763 \pm 38$ & $47.10 \pm 0.02$ & $13486 \pm 848$ & $2905 \pm 260$ \\
\hline 99 & SDSSJ113307.63+52283 & 3.744 & $46.81 \pm 0.77$ & $7358 \pm 3905$ & $1857 \pm 645$ & $46.63 \pm 0.93$ & $15605 \pm 30749$ & $2905 \pm 855$ \\
\hline 100 & SDSSJ162520.31+22583 & 3.774 & $46.91 \pm 0.03$ & $2850 \pm 146$ & $1034 \pm 47$ & $47.36 \pm 0.01$ & $10635 \pm 315$ & $1926 \pm 106$ \\
\hline 101 & SDSSJ012403.77+00443 & 3.827 & $46.73 \pm 0.07$ & $3436 \pm 329$ & $712 \pm 104$ & $47.47 \pm 0.01$ & $9418 \pm 213$ & $2578 \pm 117$ \\
\hline 102 & SDSSJ144542.75+49024 & 3.881 & $47.37 \pm 0.01$ & $2080 \pm 50$ & $403 \pm 17$ & $47.77 \pm 0.01$ & $8290 \pm 124$ & $1524 \pm 55$ \\
\hline 103 & SDSSJ132420.83+42255 & 4.042 & $46.94 \pm 0.04$ & $4155 \pm 305$ & $1225 \pm 100$ & $47.56 \pm 0.01$ & $20000 \pm 1016$ & $2905 \pm 271$ \\
\hline 104 & SDSSJ105756.28+45555 & 4.130 & $47.18 \pm 0.05$ & $3412 \pm 234$ & $57 \pm 79$ & $47.76 \pm 0.01$ & $9599 \pm 205$ & $2099 \pm 118$ \\
\hline 105 & SDSSJ095511.32+59403 & 4.364 & $47.28 \pm 0.04$ & $3347 \pm 173$ & $1112 \pm 52$ & $47.57 \pm 0.02$ & $10775 \pm 468$ & $1958 \pm 176$ \\
\hline 106 & SDSSJ083946.22+51120 & 4.408 & $46.52 \pm 0.21$ & $2339 \pm 680$ & $400 \pm 214$ & $47.14 \pm 0.05$ & $7452 \pm 961$ & $1614 \pm 452$ \\
\hline 107 & SDSSJ010619.24+00482 & 4.450 & $47.41 \pm 0.03$ & $2432 \pm 112$ & $915 \pm 37$ & $47.41 \pm 0.03$ & $7122 \pm 398$ & $2062 \pm 193$ \\
\hline 108 & SDSSJ134743.29+49562 & 4.536 & $47.14 \pm 0.25$ & $4163 \pm 964$ & $699 \pm 282$ & $47.55 \pm 0.10$ & $7979 \pm 600$ & $2448 \pm 542$ \\
\hline 109 & SDSSJ163636.92+31571 & 4.570 & $46.60 \pm 0.28$ & $2352 \pm 1058$ & $1201 \pm 339$ & $47.24 \pm 0.07$ & $8397 \pm 1327$ & $2185 \pm 574$ \\
\hline 110 & SDSSJ143835.95+43145 & 4.669 & $47.28 \pm 0.11$ & $3784 \pm 560$ & $297 \pm 168$ & $47.66 \pm 0.05$ & $9529 \pm 599$ & $2905 \pm 494$ \\
\hline 111 & SDSSJ105123.03+35453 & 4.924 & $46.99 \pm 0.04$ & $1604 \pm 110$ & $463 \pm 36$ & $47.40 \pm 0.02$ & $4798 \pm 159$ & $1764 \pm 103$ \\
\hline 112 & SDSSJ153650.25+50081 & 4.941 & $47.15 \pm 0.02$ & $1281 \pm 47$ & $1298 \pm 15$ & $47.68 \pm 0.01$ & $4974 \pm 90$ & $1487 \pm 28$ \\
\hline
\end{tabular}

Note. Column (1): sequence number; column (2): name of high- $z$ QSOs; column (3): redshift used to fit spectrum; columns (4)-(9): the profile fitting parameters of C IV (luminosity, FWHM, and peak). Subscript N and B indicate narrow and broad components, respectively. Note that the profile parameters may be problematic for QSQs with $z<1.7$ due to the incompleteness of the C IV profile.

distribution are $549.22 \mathrm{~km} \mathrm{~s}^{-1}$ and $871.34 \mathrm{~km} \mathrm{~s}^{-1}$, respectively. We note that Coatman et al. (2017) showed a more extended tail of blueshift ( $>3000 \mathrm{~km} \mathrm{~s}^{-1}$ ) based on the rest frame defined by Balmer lines, which may originate from a different definition of systemic redshift in our work and their work. We also note that the blueshift of PG and high- $z$ QSOs are $\sim 300 \mathrm{~km} \mathrm{~s}^{-1}$ and $\sim 700 \mathrm{~km} \mathrm{~s}^{-1}$,respectively. The lower blueshift for PG QSOs is correlative with the lower bolometric luminosity, which reduces the average blueshift of our sample.

We explore the relationship between the C IV velocity shift and the continuum luminosity at $5100 \AA$. Figure 4 shows that there is a weak correlation between the C IV blueshift and 

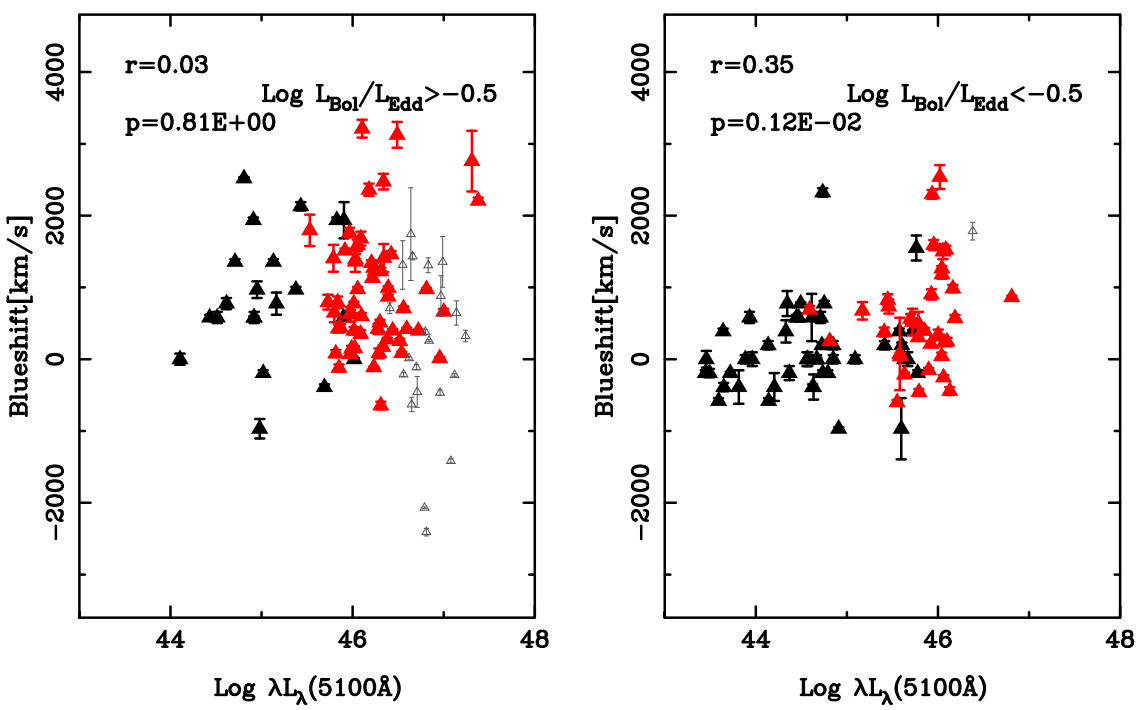

Figure 5. Relationship between the C IV velocity shift and $L\left(5100 \AA\right.$ ) in high $L_{\mathrm{Bol}} / L_{\mathrm{Edd}}$ (left) and low $L_{\mathrm{Bol}} / L_{\mathrm{Edd}}$ (right). The black and red points represent low- $z$ and high- $z$ QSOs (including Shemmer et al. 2004; Netzer et al. 2007; Assef et al. 2011; Shen \& Liu 2012), respectively. The gray points are from Jun et al. (2015). The method of calculating Spearman correlation coefficients is the same as Figure 4.

$L(5100 \AA)$ for the sample, where $r=0.23$ and $p=2.3 \times$ $10^{-3}$. The weak correlation may be due to the mixing of $L_{\mathrm{Bol}} / L_{\mathrm{Edd}}$. Figure 5 shows the relationship between the C IV blueshift and the continuum luminosity at $5100 \AA$ in different $L_{\mathrm{Bol}} / L_{\mathrm{Edd}}$ bins. We can see the correlation coefficient for low $L_{\mathrm{Bol}} / L_{\mathrm{Edd}}$ is higher than that of high $L_{\mathrm{Bol}} / L_{\mathrm{Edd}}(0.38$ versus $0.16)$. The result is consistent with that given by Shen et al. (2016). They investigated the velocity shifts of QSO emission lines from the SDSS reverberation mapping project and found that the velocity shift of C IV relative to $\mathrm{Mg}$ II has a stronger luminosity dependence than other emission lines (such as [O II], [O III], and $\mathrm{Mg}$ II). It is worth noting that our correlation test in right panel of Figure 5 agrees with Shen et al. (2016; $r \sim 0.4$ ) when considering only the objects with the continuum luminosity less than $\sim 10^{46} \mathrm{erg} \mathrm{s}^{-1}$. In this work, there are 14 radio-loud QSOs in PG QSOs and 11 high-z QSOs, respectively. We find that the correlation coefficients for the C IV blueshift and $L(5100 \AA) / L_{\mathrm{Bol}} / L_{\mathrm{Edd}}$ only increase by 0.06 after excluding the radio-loud QSOs. Therefore, radio-loud QSOs in our sample do not significantly affect our results. These radio-loud QSOs have, on average, smaller C IV blueshift $\left(132 \mathrm{~km} \mathrm{~s}^{-1}\right)$ than those of radio-quiet QSOs $\left(616 \mathrm{~km} \mathrm{~s}^{-1}\right)$, which is consistent with the result of Richards et al. (2011).

Figure 6 displays the relationship between the C IV blueshift and $L_{\mathrm{Bol}} / L_{\mathrm{Edd}}$ for the sample, where $r=0.27$ and $p=3.3 \times$ $10^{-4}$. It is found that the relation will become flat when we go to higher $L_{\mathrm{Bol}} / L_{\mathrm{Edd}}$. The possible explanation for the flat relation is that the size of BLR does not depend on the luminosity in high accretion rate QSOs (Du et al. 2018). Some studies have suggested that the accretion disk will become geometrically thick (e.g., slim disk) in the high accretion rate regime, which significantly reduces the amount of the ionizing photons reaching the BLR, and then leads to saturated luminosity (Wang \& Zhou 1999; Wang et al. 2014). The self-shadowing effect of slim disks reduces the radiation pressure and flattens the relation between the C IV blueshift and $L_{\mathrm{Bol}} / L_{\mathrm{Edd}}$ or $L(5100 \AA)$. Ge et al. (2016) estimated the intrinsic total luminosity of low- $z$ and high- $z$ QSOs based on a simple scaling factor. Such a rough calculation may not reveal the intrinsic relations of these parameters. Actually, the reradiation from dust has great

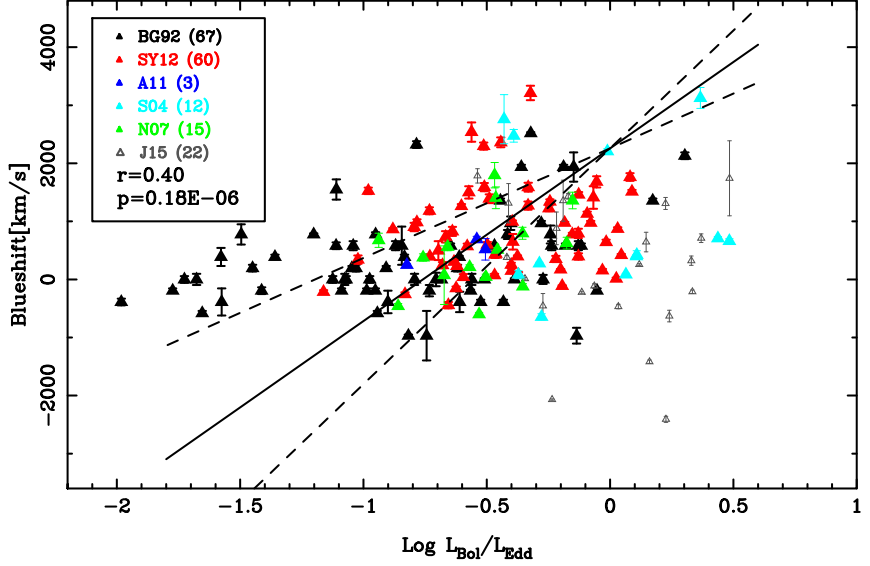

Figure 6. Relationship between the C IV velocity shift and $L_{\mathrm{Bol}} / L_{\mathrm{Edd}}$. All of the symbols and labels are the same as Figure 4. The solid line is the best fit of the Bivariate Correlated Errors and intrinsic Scatter (BCES) bisector (removing the sources from Jun et al. 2015) and the dashed lines are the best fit of the BCES bisector with $2 \sigma$ variance. $0.3 \mathrm{dex}$ is adopted as the typical error of $L_{\mathrm{Bol}} / L_{\mathrm{Edd}}$ in the fitting.

contamination to the intrinsic AGN luminosity, thus the $L_{\mathrm{Bol}} / L_{\mathrm{Edd}}$. Marconi et al. (2004) constructed a spectral energy distribution (SED) template spectrum without the infrared bump based on $\alpha$ (optical-UV spectral index) and $\alpha_{\text {ox }}$ (optical-X-ray spectral index). They derived the bolometric correction, which depends on monochromatic luminosity from the templates. For our sample, the range of luminosity at $5100 \AA$ is from $10^{43}$ to $10^{47} \mathrm{erg} \mathrm{s}^{-1}$ and the corresponding bolometric correction is between 5 and 9 . So in this work, we may overestimate the $L_{\mathrm{Bol}} / L_{\mathrm{Edd}}$, thus affecting our results. We also find an increasing correlation between the CIV blueshift and the luminosity of $5100 \AA\left(L_{\mathrm{Bol}} / L_{\mathrm{Edd}}\right)$ when we remove the sources from Jun et al. (2015) in our sample. The correlation coefficient and the probability of the null hypothesis are $0.37(0.40)$ and $1.8 \times 10^{-6}$ $\left(1.8 \times 10^{-7}\right)$, respectively. Simultaneously, we fitted the relationship between the CIV blueshift and $L_{\mathrm{Bol}} / L_{\mathrm{Edd}}$ (solid 


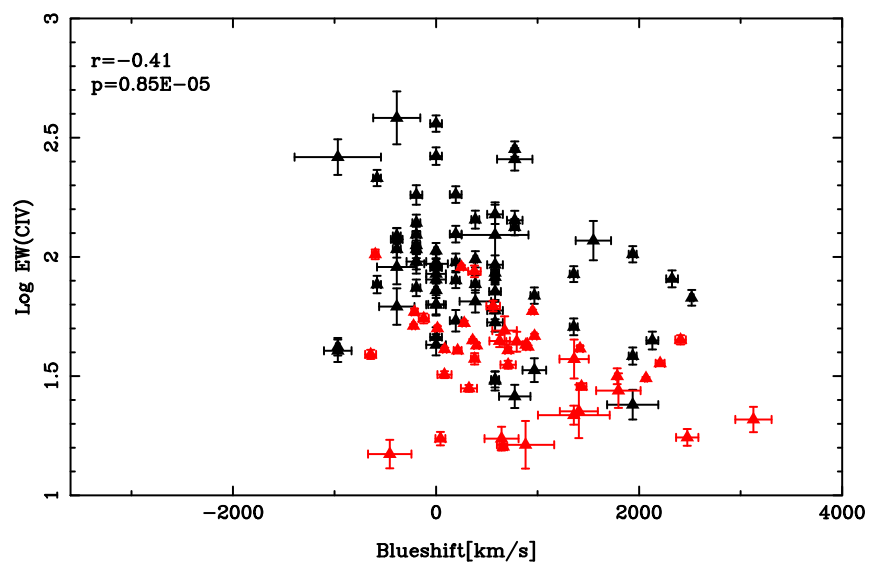

Figure 7. Relationship between the C IV velocity shift and EW(C IV). The correlation coefficients are marked at the top of the panel. PG QSOs and high- $z$ QSOs with $\mathrm{C}$ IV $\mathrm{S} / \mathrm{N}>5$ and $z>2.0$ are marked with black and red points.

line) in Figure 6. The formula for the best fit is

$$
\begin{array}{rl}
V(\mathrm{C} \mathrm{IV})=2 & 257.90( \pm 312.17)+2974.10( \pm 548.23) \\
& \times \log L_{\text {bol }} / L_{\text {Edd }} .
\end{array}
$$

The resolution of the infrared spectra from Jun et al. (2015) is the lowest $(R \sim 200)$, which can also affect the precision of $L_{\mathrm{Bol}} / L_{\mathrm{Edd}}$. In addition, Wang et al. (2011) presented a comparison of kinematics between $\mathrm{C}$ IV and $\mathrm{Mg}$ II emission lines using SDSS data. They found that the blueshift of C IV is strongly correlative with $L_{\mathrm{Bol}} / L_{\mathrm{Edd}}$, which is especially prominent in the high $L_{\mathrm{Bol}} / L_{\mathrm{Edd}}$ regime. The same result was found by Sun et al. (2018) recently, who used multi-epoch SDSS spectra to investigate the dependence of the C IV blueshift on QSO properties.

Some scenarios have been proposed to explain the physical origin of the C IV blueshift, and one of them is the orientation effect (Denney 2012). However, the scenario was excluded by Runnoe et al. (2014), who found no correlation between the $\mathrm{C}$ IV blueshift and the orientation based on a sample of radio core dominance. On the other hand, the large $L_{\mathrm{Bol}} / L_{\mathrm{Edd}}$ can cause high-blueshift emission lines, as mentioned above. In general, the QSOs with high $L_{\mathrm{Bol}} / L_{\mathrm{Edd}}$ display more apparent blueshift before approaching saturated luminosity. It indicates that the radiation pressure plays an important role in driving the shift of the peak wavelength of emission lines.

With the principal component analysis (PCA) of the low- $z$ PG sample, Boroson \& Green (1992) found that principal component 1 (PC1) is related to the relative strength of optical Fe II to $\mathrm{H} \beta$ ( $R_{\mathrm{Fe}}$, the ratio between the strength of Fe II emission and $\mathrm{H} \beta$ ) and that principal component 2 (PC2) links optical luminosity and $\alpha_{\mathrm{ox}}$. With $\mathrm{H} \beta$-based $M_{\mathrm{BH}}$, Boroson (2002) suggested that PC1 is mainly correlated with $L_{\mathrm{Bol}} / L_{\mathrm{Edd}}$ and PC2 has a strong connection with $M_{\mathrm{BH}}$ and $L_{\mathrm{Bol}} / L_{\mathrm{Edd}}$. We explore the relationship between the C IV blueshift, PC1/PC2, and $R_{\mathrm{Fe}}$ for PG QSOs. It is found that the C IV blueshift has a very weak correlation with $\mathrm{PC} 1$ or $R_{\mathrm{Fe}}$, but has a mediumstrength correlation with PC2. The correlation coefficients are $-0.11,0.15$, and -0.33 for $\mathrm{PC} 1, R_{\mathrm{Fe}}$, and $\mathrm{PC} 2$, respectively. The relation between $\mathrm{PC} 1 / \mathrm{PC} 2$ and $L_{\mathrm{Bol}} / L_{\mathrm{Edd}} / M_{\mathrm{BH}}$ needs to be investigated with more reliable measurements of $M_{\mathrm{BH}}$ in QSOs.

In order to explore the relationship between EW (C IV) and the $\mathrm{C}$ IV blueshift, We choose a subsample containing 67 PG
QSOs and 43 high- $z$ QSOs from our sample. These high- $z$ QSOs have $\mathrm{C}$ IV emission line $\mathrm{S} / \mathrm{N}>5$ and $z>2$. Figure 7 shows the relationship between EW (C IV) and the C IV blueshift. A medium-strength correlation is found between them $(r=-0.41)$, which is consistent with the wind-disk model of BLRs given by Richards et al. (2011). Higher $L_{\mathrm{Bol}} / L_{\mathrm{Edd}}$ may lead to the formation of a wind component. If the BLR is dominated by the wind component, broad emission lines will show the blueshift and their strength will be suppressed (Richards et al. 2011).

Alternatively, Shemmer \& Lieber (2015) pointed out that the lowest scatter relationship is not continuum versus EW (C IV), but rather $\mathrm{H} \beta$-based $L_{\mathrm{Bol}} / L_{\mathrm{Edd}}$, i.e., the modified Baldwin effect (MBE). The MBE can also be used to explain the relationship between the total EW (C IV) and the C IV blueshift. As $L_{\mathrm{Bol}} / L_{\mathrm{Edd}}$ changes, there are expected changes in the thickness of the inner accretion disk, which may well affect the ionization of the BLR and a disk wind. However, weak-line QSOs deviating considerably from the classical Baldwin effect and MBE in Shemmer \& Lieber (2015) reveal that the relationship between the profile and EW (C IV) may not only depend on $L_{\mathrm{Bol}} / L_{\mathrm{Edd}}$, but also additional physical properties (such as BLR geometry, density, and metallicity). Furthermore, if objects are viewed pole-on, then these objects will exhibit a narrower line width (Shen \& Ho 2014), but actually lower $L_{\mathrm{Bol}} / L_{\mathrm{Edd}}$. The orientation effect will result in a larger deviation from MBE for weak-line QSOs. Broadly speaking, the orientation effect cannot be the main explanation of the EW change. In addition, we find that some objects with high $L_{\mathrm{Bol}} / L_{\mathrm{Edd}}$ deviate from the relationship between $L_{\mathrm{Bol}} / L_{\mathrm{Edd}}$ and blueshift (Figure 6), suggesting that these objects might suffer from the orientation bias.

The relationship between EW (C IV) and the C IV blueshift also supports the classical Baldwin effect originating from the softening of high-energy photons (Netzer et al. 1992; Korista et al. 1998; Dietrich et al. 2002). As suggested by Richards et al. (2011), the reduction of the number of high-energy photons that can ionize gas is beneficial to the formation of the wind component, thus enhancing the blueshift and reducing the EW. The wind component is not separated from the disk component in our fitting model. Therefore we are not sure that whether the Baldwin effect is triggered by the disappearance or weakness of the wind component.

\subsection{CIV-based $M_{B H}$ Correction with the CIV Blueshift}

It is found that the C IV-based $M_{\mathrm{BH}}$ is biased with respect to that based on $\mathrm{H} \beta$ (Bian et al. 2012; Shen \& Liu 2012). This bias is suggested to be corrected by the C IV blueshift (Coatman et al. 2017; Jun et al. 2017). Figure 8 shows the relationship between the $M_{\mathrm{BH}}$ difference (C IV-based $M_{\mathrm{BH}}$ and $\mathrm{H} \beta$-based $M_{\mathrm{BH}}$ ) and the C IV blueshift. It is found that there is a mediumstrong correlation between them with the correlation coefficient of $r=0.35$ and the probability of the null hypothesis of $p=$ $2.2 \times 10^{-4}$. The black line is the best fit of the BCES bisector. The formula is

$$
\begin{aligned}
\log \left(M_{\mathrm{BH}}(\mathrm{C} \mathrm{IV} / \mathrm{H} \beta)\right)= & -0.34( \pm 0.09)+6.31( \pm 1.57) \\
& \times 10^{-4} V(\mathrm{C} \mathrm{IV})
\end{aligned}
$$

The red and green dashed lines in Figure 8 are from Jun et al. (2017) and Coatman et al. (2017), respectively. We correct the C IV-based $M_{\mathrm{BH}}$ according our best fit and find that the mean 


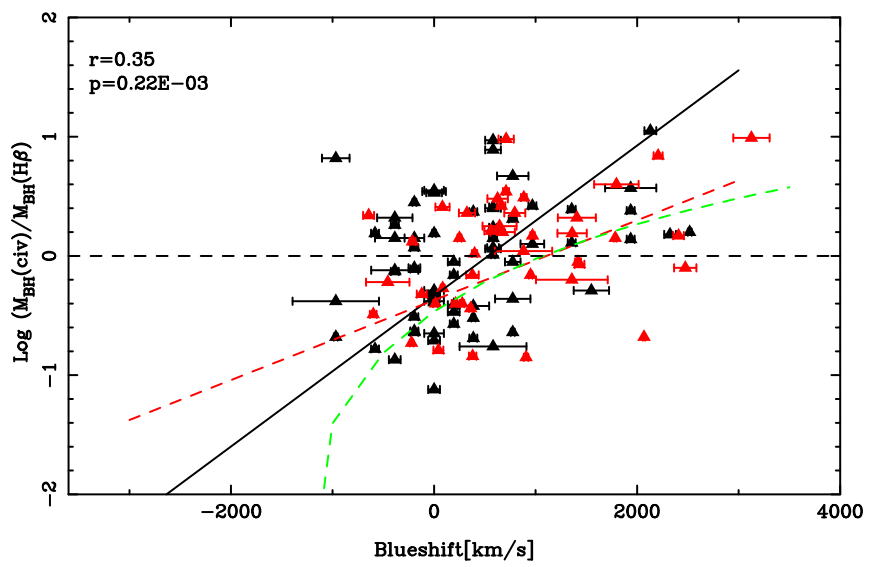

Figure 8. Effect of the $\mathrm{C}$ IV velocity shift on the difference between $M_{\mathrm{BH}}$ based on $\mathrm{C}$ IV and $\mathrm{H} \beta$. The black solid line is the best fit of the BCES bisector. Red and green dashed lines are the relations from Jun et al. (2017) and Coatman et al. (2017). The correlation coefficients are listed at the top of the panel. The objects used for the analysis are the same as those in Figure 7.

value and the dispersion of the $M_{\mathrm{BH}}$ difference are 0.012 and 0.48 dex, respectively, which shows that some other parameters are need to be considered in the calculation of $M_{\mathrm{BH}}$ from the C IV line.

Compared to $\mathrm{H} \beta$-based $M_{\mathrm{BH}}$, many previous studies have shown that the C IV-based single-epoch SMBH mass usually exhibits significant scatter (Baskin \& Laor 2004b; Richards et al. 2011; Bian et al. 2012; Denney 2012; Shen \& Liu 2012). Coatman et al. (2017) used a high- $z$ AGN sample $(1.5<z<4.0)$ to investigate the relationship between the C IV blueshift and C IV-based $M_{\mathrm{BH}}$. They found that C IV-based $M_{\mathrm{BH}}$ may be a factor 5 to 10 larger than Balmer-line-based $M_{\mathrm{BH}}$ at the C IV blueshift of more than $3000 \mathrm{~km} \mathrm{~s}^{-1}$ and gave an empirical correction for the CIV-based $M_{\mathrm{BH}}$ based on the relation between the C IV blueshift and the ratio of C IV FWHM to $\mathrm{H} \alpha$ FWHM. In contrast, Mejía-Restrepo et al. (2018) used a larger sample (including the objects from Coatman et al. 2017) and showed that the relation between FWHM $(\mathrm{C} \mathrm{IV} / \mathrm{H} \alpha) /$ FWHM (C IV $/ \mathrm{Mg}$ II) and the C IV blueshift is driven by the relation between the C IV blueshift and C IV FWHM, which suggested that the empirical correction constructed by the $\mathrm{CIV}$ blueshift and Balmer-line width for C IV-based single-epoch $M_{\mathrm{BH}}$, therefore, the $L_{\mathrm{Bol}} / L_{\mathrm{Edd}}$, is limited. In addition, they also found that there is no connection between $\mathrm{Mg}$ II and $\mathrm{C}$ IV profiles according to the PCA analysis, suggesting that highionization (C IV) and low-ionization ( $\mathrm{H} \alpha, \mathrm{Mg}$ II) lines are distinct.

We find the C IV blueshift is indeed related to the ratio of $M_{\mathrm{BH}}$ (Figure 8). However, our correction for C IV-based singleepoch $M_{\mathrm{BH}}$ is limited considering the large system error of $M_{\mathrm{BH}}$, which also seems to support the conclusion given by Mejía-Restrepo et al. (2018) that solely using the C IV line width may not get the reliable virialized $M_{\mathrm{BH}}$. Line peak ratios need to be considered (Runnoe et al. 2013; Brotherton et al. 2015). To understand how to improve the accuracy of C IVbased single-epoch $M_{\mathrm{BH}}$, large samples including low- and high- $z$ AGNs are required for future analysis.

The difference of the results among Coatman et al. (2017), Mejía-Restrepo et al. (2018), and our work may originate from the sample selection. The similarity about the samples is that all of these studies use the sample of Shen \& Liu (2012). However, most of the objects in Shen \& Liu (2012) are $z<1.7$, which leads the incompleteness of the C IV profiles in the blue waveband. Therefore, the profile parameters, such as FWHM and EW may be inaccurate for these objects. We select objects with $z>2.0$ to avoid the issue and investigate the effects of the C IV blueshift on C IV-based single-epoch $M_{\mathrm{BH}}$ in Section 3.2. In addition, a difference in the samples is the utilization of PG QSOs $(z<0.5)$ in this work. PG QSOs have lower bolometric luminosity than high- $z$ QSOs $(z>2.0)$, which allow us to expand our results to the end of low luminosity.

\section{Conclusion}

For a collected sample of low $z$, and high- $z$ QSOs, spectral decomposition is used to investigate the blueshift of C IV broad emission line relative to systemic, which is defined by [O III] $\lambda 5007$. The results are as follows.

(1) It is confirmed that a blueshift exists for the highionization $\mathrm{C}$ IV broad emission line in the rest frame defined by the narrow [O III] line or $\mathrm{H} \beta$. It is found that there is a medium-strong positive correlation between the C IV blueshift and the luminosity of continuum or $L_{\mathrm{Bol}} / L_{\mathrm{Edd}}$, and a medium negative relationship between the CIV blueshift and EW(C IV). These results are consistent with the picture that radiation pressure is correlative to the C IV blueshift.

(2) There is a medium-strong correlation between the ratio of C IV-based $M_{\mathrm{BH}}$ to $\mathrm{H} \beta$-based $M_{\mathrm{BH}}$ and the $\mathrm{C}$ IV blueshift. This relationship depends on the accurate systemic redshift and the $\mathrm{H} \beta$-based $M_{\mathrm{BH}}$. A larger sample is needed to investigate this relationship.

We would like to thank M. S. Brotherton for the useful discussions. This work is supported by the National Key Research and Development Program of China (No. 2017YFA0402703). This work has been supported by the National Science Foundations of China (Nos. 11373024, 11233003, and 11873032).

\section{Appendix}

\section{ORCID iDs}

Xue Ge $\mathbb{D}$ https://orcid.org/0000-0002-8348-2783

Bi-Xuan Zhao (i) https://orcid.org/0000-0002-5413-3680

Wei-Hao Bian (iD https://orcid.org/0000-0002-2121-8960

\section{References}

Abazajian, K. N., Adelman-McCarthy, J. K., Agüeros, M. A., et al. 2009, ApJS, 182, 543

Anand, M. Y., Kagali, B. A., \& Murthy, J. 2009, BASI, 37, 1

Assef, R. J., Denney, K. D., Kochanek, C. S., et al. 2011, ApJ, 742, 93

Baskin, A., \& Laor, A. 2004a, MNRAS, 350, L31

Baskin, A., \& Laor, A. 2004b, MNRAS, 356, 1029

Bian, W.-H., Fang, L.-L., Huang, K.-L., \& Wang, J. M. 2012, MNRAS, 427, 2881

Bian, W.-H., He, Z.-C., Green, R., et al. 2016, MNRAS, 456, 4081

Boroson, A. T., \& Green, R. F. 1992, ApJS, 80, 109

Boroson, T. A. 2002, ApJ, 565, 78

Brandt, W. N., Laor, A., \& Wills, B. J. 2000, ApJ, 528, 637

Brotherton, M. S., Runnoe, J. C., Shang, Z., \& DiPompeo, M. A. 2015, MNRAS, 451, 1290 
Brotherton, M. S., Wills, B. J., Steidel, C. C., \& Sargent, W. L. W. 1994, ApJ, 423, 131

Cardelli, J. A., Clayton, G. C., \& Mathis, J. S. 1989, ApJ, 345, 24

Coatman, L., Hewett, P. C., Banerji, M., \& Richards, G. T. 2017, MNRAS, 465,2120

Collin, S., Kawaguchi, T., Peterson, B. M., \& Vestergaard, M. 2006, A\&A, 456, 75

Denney, K. D. 2012, ApJ, 759, 44

Dietrich, M., Hamann, F., Shields, J. C., et al. 2002, ApJ, 581, 912

Du, P., Zhang, Z. X., Wang, K., et al. 2018, ApJ, 856, 6

Gaskell, C. M. 1982, ApJ, 263, 79

Ge, X., Bian, W.-H., Jiang, X.-L., Liu, W.-S., \& Wang, X.-F. 2016, MNRAS, 462,966

Gunn, J. E., Siegmund, W. A., Mannery, E. J., et al. 2006, AJ, 131, 2332

Hewett, P. C., \& Wild, V. 2010, MNRAS, 405, 2302

Kaspi, S., Smith, P. S., Netzer, H., et al. 2000, ApJ, 533, 631

Jun, H. D., Im, M., Kim, D., \& Stern, D. 2017, ApJ, 838, 41

Jun, H. D., Im, M., Lee, H. M., et al. 2015, ApJ, 806, 109

Korista, K., Baldwin, J., \& Ferland, G. 1998, ApJ, 507, 24

Laor, A., \& Brandt, W. N. 2002, ApJ, 569, 641

Leighly, K. M. 2004, ApJ, 611, 125

Marconi, A., Risaliti, G., Gilli, R., et al. 2004, MNRAS, 351, 169

Marziani, P., Sulentic, J. W., Dultzin-Hacyan, D., Calvani, M., \& Moles, M. 1996, ApJS, 104, 37

McLure, R. J., \& Jarvis, M. J. 2002, MNRAS, 337, 109

Mejía-Restrepo, J. E., Trakhtenbrot, B., Lira, P., \& Netzer, H. 2018, MNRAS, 478, 1929

Murray, N., Chiang, J., Grossman, S. A., \& Voit, G. M. 1995, ApJ, 451, 498

Netzer, H., Laor, A., \& Gondhalekar, P. M. 1992, MNRAS, 254, 15

Netzer, H., Lira, P., Trakhtenbrot, B., Shemmer, O., \& Cury, I. 2007, ApJ, 671,1256
Onken, C. A., \& Kollmeier, J. A. 2008, ApJ, 689, L13

Pâris, I., Petitjean, P., Ross, N. P., et al. 2016, A\&A, 597, A79

Proga, D., Stone, J. M., \& Kallman, T. R. 2000, ApJ, 543, 686

Richards, G. T., Kruczek, N. E., Gallagher, S. C., et al. 2011, AJ, 141, 167

Richards, G. T., Vanden Berk, D. E., Reichard, T. A., et al. 2002, AJ, 124,1

Runnoe, J. C., Brotherton, M. S., DiPompeo, M. A., \& Shang, Z. 2014, MNRAS, 438, 3263

Runnoe, J. C., Brotherton, M. S., Shang, Z., \& DiPompeo, M. A. 2013, MNRAS, 434, 848

Schmidt, M., \& Green, R. F. 1983, ApJ, 269, 352

Shemmer, O., \& Lieber, S. 2015, ApJ, 805, 124

Shemmer, O., Netzer, H., Maiolino, R., et al. 2004, ApJ, 614, 547

Shen, Y., Brandt, W. N., Richards, G. T., et al. 2016, ApJ, 831, 7

Shen, Y., \& Ho, L. C. 2014, Natur, 513, 210

Shen, Y., \& Liu, X. 2012, ApJ, 753, 125

Shen, Y., Richards, G. T., Strauss, M. A., et al. 2011, ApJS, 194, 45

Shi, Y., Rieke, G. H., Ogle, P. M., et al. 2014, ApJS, 214, 23

Sulentic, J. W., Marziani, P., \& Dultzin-Hacyan, D. 2000, ARA\&A, 38, 521

Sun, M. Y., Xue, Y. Q., Richards, G. T., et al. 2018, ApJ, 854, 128

Tytler, D., \& Fan, X. M. 1992, ApJS, 79, 1

Vanden Berk, D. E., Richards, G. T., Bauer, A., et al. 2001, AJ, 122, 549

Vestergaard, M., \& Peterson, B. M. 2006, ApJ, 641, 689

Wang, H. Y., Wang, T., Zhou, H., et al. 2011, ApJ, 738, 85

Wang, J. M., Du, P., Brotherton, M. S., et al. 2017, NatAs, 1, 775

Wang, J. M., Qiu, J., Du, P., \& Ho, L. C. 2014, ApJ, 797, 65

Wang, J. M., \& Zhou, Y. Y. 1999, ApJ, 516, 420

Wills, B. J., Brotherton, M. S., Fang, D., Steidel, C. C., \& Sargent, W. L. W. 1993, ApJ, 415, 563

Wu, J., Vanden Berk, D. E., Brandt, W. N., et al. 2009, ApJ, 702, 767

York, D. G., Adelman, J., Anderson, J. E., Jr., et al. 2000, AJ, 120, 1579 